OPEN ACCESS

Edited by:

Stephen J. Pandol,

Cedars-Sinai Medical Center,

United States

Reviewed by:

Gabriel Samasca,

Iuliu Hațieganu University of Medicine

and Pharmacy, Romania

Giuseppe Losurdo,

University of Bari Medical School, Italy

*Correspondence:

Péter Hegyi

hegyi2009@gmail.com

tThese authors have contributed equally to this work

Specialty section: This article was submitted to

Gastrointestinal Sciences,

a section of the journal

Frontiers in Physiology

Received: 19 April 2019 Accepted: 31 October 2019 Published: 19 November 2019

Citation:

Szakács Z, Gede N, Gyöngyi Z,

Solymár M, Csupor D, Eröss $B$, Vincze Á, Mikó A, Vasas A, Szapáry L,

Dobszai D, Balikó V, Hágendorn R, Hegyi $P$ and Bajor J (2019) A Call for

Research on the Prognostic Role of Follow-Up Histology in Celiac Disease:

A Systematic Review.

Front. Physiol. 10:1408.

doi: 10.3389/fphys.2019.01408

\section{A Call for Research on the Prognostic Role of Follow-Up Histology in Celiac Disease: A Systematic Review}

\author{
Zsolt Szakács ${ }^{1,2}$, Noémi Gede ${ }^{1}$, Zoltán Gyöngyi ${ }^{3}$, Margit Solymár ${ }^{1}$, Dezsö Csupor ${ }^{4}$, \\ Bálint Eröss ${ }^{1,2}$, Áron Vincze ${ }^{5}$, Alexandra Mikó ${ }^{1}$, Andrea Vasas ${ }^{3}$, László Szapáry ${ }^{1,6}$, \\ Dalma Dobszai ${ }^{1}$, Viktória Balikó ${ }^{1}$, Roland Hágendorn ${ }^{5}$, Péter Hegyi ${ }^{1,2 \star t}$ and Judit Bajor ${ }^{5 \dagger}$ \\ ${ }^{1}$ Institute for Translational Medicine, Medical School, University of Pécs, Pécs, Hungary, ${ }^{2}$ Szentágothai Research Centre, \\ Medical School, University of Pécs, Pécs, Hungary, ${ }^{3}$ Department of Public Health Medicine, Medical School, University of \\ Pécs, Pécs, Hungary, ${ }^{4}$ Department of Pharmacognosy, University of Szeged, Szeged, Hungary, ${ }^{5}$ Division of \\ Gastroenterology, First Department of Medicine, Medical School, University of Pécs, Pécs, Hungary, ${ }^{6}$ Department of \\ Interventional Cardiology, Heart Institute, University of Pécs, Pécs, Hungary
}

Background: Convincing evidence is lacking on the benefit of follow-up biopsy in celiac disease. Regardless, achieving mucosal recovery (MR) has remained a desirable goal of therapy. We aimed to conduct a systematic review to determine whether MR is a protective factor and persisting villous atrophy (PVA) has negative consequences on long-term outcomes of celiac disease.

Methods: Seven databases were searched for articles discussing celiac patients subjected to a gluten-free diet who had a follow-up biopsy, and clinical and laboratory characteristics were reported by follow-up histology (MR vs. PVA). Outcomes included clinical symptoms, mortality, malignant tumors, nutritional parameters, and metabolic bone disease. Comparative and descriptive studies were included. Since data proved to be ineligible for meta-analysis, the evidence was synthesized in a systematic review.

Results: Altogether, 31 studies were eligible for systematic review. Persisting symptoms were more frequently associated with PVA than with MR, although a lot of symptom-free patients had PVA and a lot of symptomatic patients achieved MR. PVA might be a risk factor of lymphomas, but mortality and the overall rate of malignant tumors seemed independent of follow-up histology. Patients with PVA tended to develop metabolic bone disease more often, although fracture risk remained similar in the groups except in hip fractures of which PVA was a risk factor. Reports on nutritional markers are only anecdotal.

Conclusions: The limited evidence calls for high-quality prospective cohort studies to be arranged to clarify the exact role of follow-up histology in celiac disease.

Keywords: celiac disease, mucosal recovery, persistent villous atrophy, follow-up biopsies, gluten-free diet 


\section{INTRODUCTION}

Celiac disease $(\mathrm{CeD})$ is a systemic disorder with an increasing worldwide prevalence of $\sim 1 \%$ (Di Sabatino and Corazza, 2009; Catassi et al., 2014). In CeD, the immune-mediated destruction of small intestinal villous architecture is triggered by dietary gluten (Reilly et al., 2017).

\section{Rationale of the Study}

Although the value of intestinal biopsy at diagnosis is beyond dispute (Bai et al., 2013; Rubio-Tapia et al., 2013; Ludvigsson et al., 2014), the role of follow-up biopsy is a matter of controversy (Pekki et al., 2017). While the restitution of intestinal villi is expected on a strict gluten-free diet, the mucosa fails to restore entirely in a considerable fraction of patients (Szakacs et al., 2017). Recent guidelines recommend a followup biopsy if signs and symptoms persist or relapse despite strict adherence to a gluten-free diet (Bai et al., 2013; RubioTapia et al., 2013; Ludvigsson et al., 2014). However, reports proposed that neither the resolution of symptoms (Bardella et al., 2007; Biagi et al., 2014; Fang et al., 2017; Mahadev et al., 2017) nor a strict dietary adherence (Szakacs et al., 2017) guarantees mucosal recovery (MR). One might expect that $\mathrm{CeD}$ patients with persistent villous atrophy (PVA) experience a less favorable disease course than those achieving MR (Haines et al., 2008), although convincing evidence is lacking. Yet, achieving $\mathrm{MR}$ has remained a desirable goal in $\mathrm{CeD}$. The importance of the topic roots in the burden imposed by the endoscopic procedures and duodenal histological sampling as well as in the subsequent clinical decisions made upon followup histology.

\section{Objective of the Study}

With this systematic review, we aimed to be the first who systematically collect all available evidence on the impact of follow-up histology (MR vs. PVA) on disease characteristics and clinical course of CeD.

\section{Research Question}

Are CeD patients who have not achieved MR exposed to adverse clinical outcomes more frequently than those patients who have achieved MR?

\section{METHODS}

\section{Study Design}

This systematic review was reported in accordance with the Preferred Reporting Items for Systematic reviews and MetaAnalyses 2009 (Moher et al., 2009).

Abbreviations: SF-36, 36-Item Short Form Health Survey; CeD, celiac disease; GSRS, Gastrointestinal Symptom Rating Scale; HR, hazard ratio; IBS, irritable bowel syndrome; MR, mucosal recovery; NS, non-significant; OR, odds ratio; PGWB, Psychological General Well-Being Index; PVA, persistent villous atrophy.

\section{Participants and Exposure}

Participants of this systematic review include $\mathrm{CeD}$ patients subjected to a gluten-free diet. The exposure of interest is the follow-up duodenal histology assessed as either MR or PVA.

\section{Systematic Review Protocol}

The study protocol was registered a priori on PROSPERO under CRD42017069815.

\section{Search Strategy}

We performed a systematic literature search in MEDLINE (via PubMed), Embase, Cochrane Controlled Register of Trials (CENTRAL), Web of Science, Scopus, WHO Global Index Medicus, and ClinicalTrials.gov from inception up to 14th September 2019 for relevant articles. Free-text terms and Medical Subject Headings were combined into a query, as follows: celiac AND ("mucosal recovery" $O R$ "mucosal healing" OR "mucosal atrophy" OR "intestinal atrophy" OR "duodenal atrophy" $O R$ "villous atrophy" $O R$ "persistent mucosal damage" OR "follow-up biopsy" OR "followup duodenal biopsy" OR "follow-up intestinal biopsy" $O R$ "follow-up small intestinal biopsy" OR "follow-up histology" OR "repeated biopsy" $O R$ "repeated histology" $O R$ "control biopsy" OR "control histology"). No filters were imposed upon the search.

Relevant cited articles were explored by reviewing the reference lists of included papers. Citing articles were identified with Google Scholar.

\section{Data Sources, Study Selection, and Data Extraction Eligibility Criteria}

Eligible papers discussed CeD patients subjected to a gluten-free diet with an available record of duodenal follow-up histology, and reported on disease and patients characteristics by followup histology. Analytical and descriptive full-text articles or conference abstracts but not case studies were included without language restriction to reduce publication bias.

In our previous meta-analysis, we experienced a substantial variance in the definition of MR across studies (Szakacs et al., 2017). In this systematic review, we defined MR as the resolution of villous atrophy at follow-up biopsy assessed as "non-atrophic" histology based on crypt height:villous depth ratio, Marsh grades 0-2, Marsh-Oberhuber grades 0-2, or Corazza-Villanacci grade A; while PVA was defined as "atrophic" histology, Marsh grade 3, Marsh-Oberhuber grade 3, or Corazza-Villanacci grade B1B2 (Marsh, 1992; Oberhuber et al., 1999; Corazza and Villanacci, 2005).

Disease and patients characteristics included signs and symptoms, vitamin and mineral levels, anemia, body mass index, metabolic bone disease, malignant tumors and other co-morbid conditions, and long-term mortality.

\section{Selection and Data Collection}

Records were combined in a reference manager software (EndNote X7.4, Clarivate Analytics, Philadelphia, PA, the USA) to remove duplicates and overlapping database content. Then, 
the standard three-step selection was performed by title, abstract and full-texts. Each step was carried out by two investigators in duplicate. Discrepancies were resolved by third-party arbitration. K-statistics was used to measure the agreement between the investigators after each step.

Numeric and text data were extracted by two investigators onto a pre-defined Excel sheet, discrepancies were resolved by consensus. Although we contacted the authors of original studies for further raw data via email, we discarded these data from the systematic review when we realized that the material is ineligible for meta-analysis due to several reasons (as detailed later).

\section{Design of the Studies Included and Quality Assessment}

First, the design of the included papers was identified. Then, quality indicators were chosen based on the Quality in Prognostic Studies tool (Hayden et al., 2006), as follows:

- way of recruitment,

- diagnosis of CeD (only biopsy-verified cases or not),
- the recency of diagnosis (newly diagnosed patients or treated patients were included),

- representativeness of study population to the general $\mathrm{CeD}$ population (based on the inclusion and exclusion criteria of the individual studies),

- timing of follow-up biopsy (taken prospectively after enrolment or earlier),

- time elapsed between the diagnosis of $\mathrm{CeD}$ and the followup biopsy, and that between the follow-up biopsy and the measurement of outcomes,

- definition of PVA (histological classification),

- biopsy sampling site,

- timing of outcome assessment (prospectively after enrolment or earlier),

- definitions of outcomes (with cut-off values if applicable),

- blinding,

- adherence to a gluten-free diet (strict or not), and

- statistical considerations (the analysis directly compared the clinical characteristics by MR and PVA or not and the analysis was adjusted for reasonable confounding factors or not).

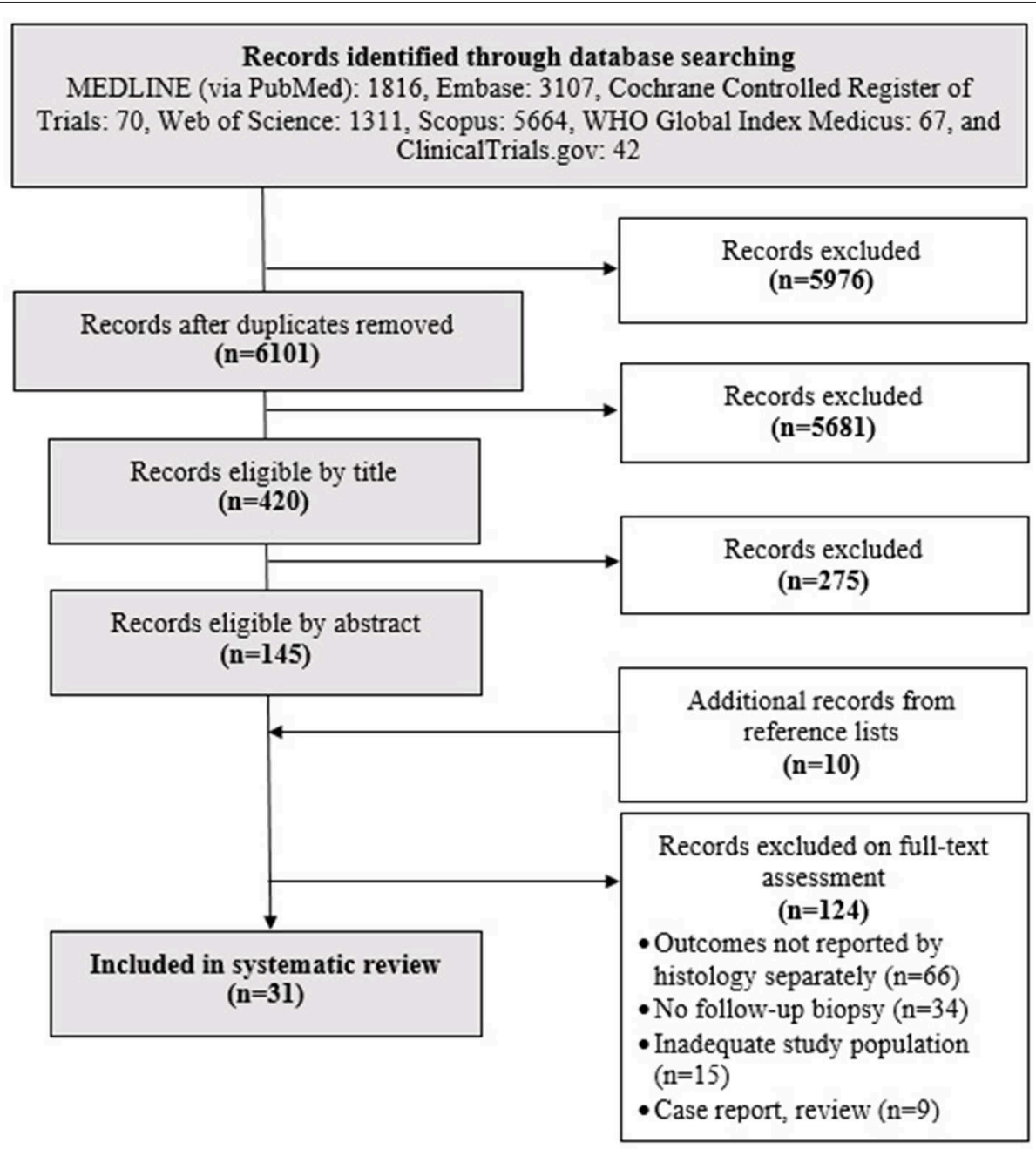

FIGURE 1 | Flowchart of the search and selection process. 
TABLE 1 | Characteristics of the studies included.

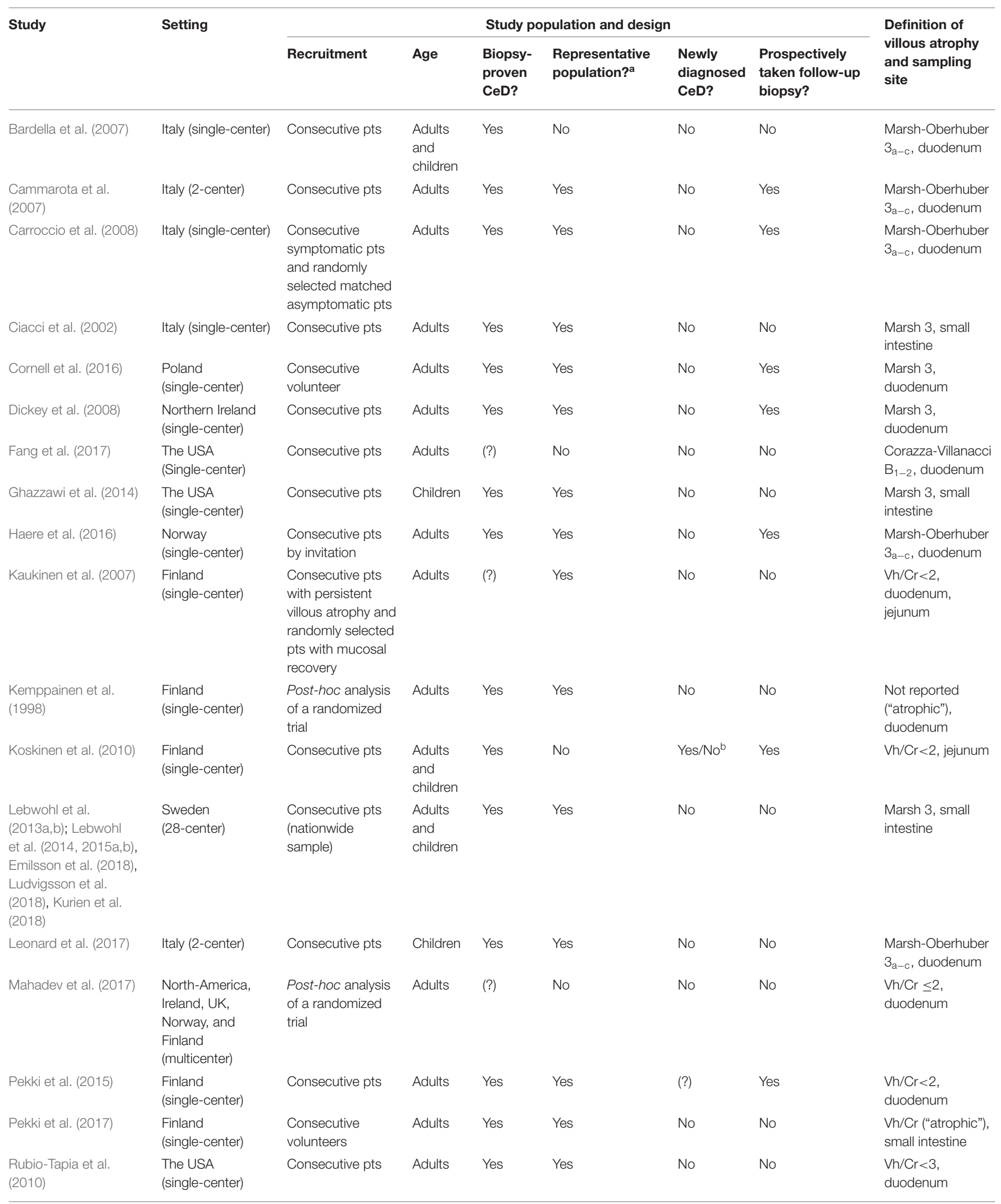


TABLE 1 | Continued

\begin{tabular}{|c|c|c|c|c|c|c|c|c|}
\hline \multirow[t]{2}{*}{ Study } & \multirow[t]{2}{*}{ Setting } & \multicolumn{6}{|c|}{ Study population and design } & \multirow{2}{*}{$\begin{array}{l}\text { Definition of } \\
\text { villous atrophy } \\
\text { and sampling } \\
\text { site }\end{array}$} \\
\hline & & Recruitment & Age & $\begin{array}{l}\text { Biopsy- } \\
\text { proven } \\
\text { CeD? }\end{array}$ & $\begin{array}{l}\text { Representative } \\
\text { population? }\end{array}$ & $\begin{array}{l}\text { Newly } \\
\text { diagnosed } \\
\text { CeD? }\end{array}$ & $\begin{array}{l}\text { Prospectively } \\
\text { taken follow-up } \\
\text { biopsy? }\end{array}$ & \\
\hline Selby et al. (1999) & $\begin{array}{l}\text { Australia } \\
\text { (single-center) }\end{array}$ & $\begin{array}{l}\text { Symptomatic and } \\
\text { asymptomatic pts } \\
\text { from a previous } \\
\text { food-intolerance } \\
\text { study }\end{array}$ & Adults & Yes & Yes & No & No & $\begin{array}{l}\text { Vh/Cr }<2 \\
\text { duodenum }\end{array}$ \\
\hline Souroujon et al. (1982) & $\begin{array}{l}\text { Israel } \\
\text { (single-center) }\end{array}$ & Consecutive pts & $\begin{array}{l}\text { Adults } \\
\text { and } \\
\text { Children }\end{array}$ & Yes & Yes & Yes & Yes & $\begin{array}{l}\text { Not reported } \\
\text { ("atrophic"), small } \\
\text { intestine }\end{array}$ \\
\hline Thornquist et al. (1993) & $\begin{array}{l}\text { Norway } \\
\text { (single-center) }\end{array}$ & $\begin{array}{l}\text { Consecutive pts } \\
\text { by invitation }\end{array}$ & Adults & Yes & Yes & No & Yes/Noc & $\begin{array}{l}\text { Alexander III-IV, } \\
\text { jejunum }\end{array}$ \\
\hline Tuire et al. (2012) & $\begin{array}{l}\text { Finland } \\
\text { (single-center) }\end{array}$ & $\begin{array}{l}\text { Consecutive } \\
\text { volunteers }\end{array}$ & Adults & Yes & Yes & No & Yes & $\begin{array}{l}\text { Marsh 3, } \\
\text { duodenum }\end{array}$ \\
\hline $\begin{array}{l}\text { Valdimarsson et al. } \\
\text { (1994) }\end{array}$ & $\begin{array}{l}\text { Sweden } \\
\text { (single-center) }\end{array}$ & $\begin{array}{l}\text { Consecutive pts } \\
\text { with persistent } \\
\text { villous atrophy and } \\
\text { matched pts with } \\
\text { mucosal recovery }\end{array}$ & Adults & $(?)$ & Yes & No & No & $\begin{array}{l}\text { Alexander III-IV, } \\
\text { small intestine }\end{array}$ \\
\hline Walters et al. (1995) & UK (single center) & $\begin{array}{l}\text { Consecutive pts } \\
\text { by invitation }\end{array}$ & Adults & Yes & No & No & Yes/Noc & $\begin{array}{l}\text { Not reported } \\
\text { ("atrophic"), small } \\
\text { intestine }\end{array}$ \\
\hline
\end{tabular}

a Study population is considered representative to the average CeD population if the study avoided inappropriate exclusions.

${ }^{b}$ The study observed a group of newly diagnosed CeD patients for the short-term and a group of followed-up patients for the long-term.

${ }^{c}$ Not all patients were newly diagnosed.

(?) indicates uncertainty. $\mathrm{CeD}$, celiac disease; pts, patients; $\mathrm{Vh} / \mathrm{Cr}$, villous height/crypt depth ratio.

\section{RESULTS}

\section{Study Selection and Characteristics}

After careful search and selection (Figure 1), 31 papers were included in the systematic review (Souroujon et al., 1982; Thornquist et al., 1993; Valdimarsson et al., 1994; Walters et al., 1995; Kemppainen et al., 1998; Selby et al., 1999; Ciacci et al., 2002; Bardella et al., 2007; Cammarota et al., 2007; Kaukinen et al., 2007; Carroccio et al., 2008; Dickey et al., 2008; Koskinen et al., 2010; Rubio-Tapia et al., 2010; Tuire et al., 2012; Lebwohl et al., 2013a,b, 2014, 2015a,b; Ghazzawi et al., 2014; Pekki et al., 2015, 2017; Cornell et al., 2016; Haere et al., 2016; Fang et al., 2017; Leonard et al., 2017; Mahadev et al., 2017; Emilsson et al., 2018; Kurien et al., 2018; Ludvigsson et al., 2018) (Table 1). Cohen's $\kappa$ representing interreviewer agreement was 0.72 (substantial), 0.79 (substantial), and 0.92 (almost perfect) for selection by title, abstract, and full-text; respectively.

Out of the 31 papers, eight retrieved data on different outcomes from the Swedish CeD registry; therefore, study populations partially overlap (Lebwohl et al., 2013a,b, 2014, 2015a,b; Emilsson et al., 2018; Kurien et al., 2018; Ludvigsson et al., 2018).

\section{Highlights of the Last Five Years}

In this subchapter, we summarize the findings of the studies which investigated the prognostic role of follow-up histology and were published after January 2015 (Lebwohl et al., 2013a, 2015a; Pekki et al., 2015, 2017; Haere et al., 2016; Fang et al., 2017; Mahadev et al., 2017; Emilsson et al., 2018; Kurien et al., 2018; Ludvigsson et al., 2018). Eight studies were conducted in Scandinavia, one in the USA, and there was another multicenter study from Europe and North-America. Evidence from univariate analysis suggested a borderline association of PVA with persisting symptoms (OR $=1.656$ with CI: $0.949-$ $2.889 ; p=0.076$ favoring mucosal recovery) (Fang et al., 2017), which was not confirmed by co-variate-adjusted analysis of the baseline cohort of patients of a multicenter randomized trial on symptomatic CeD patients (Mahadev et al., 2017). Conclusions from three Scandinavian studies on symptom and well-being scores corroborated these findings (Pekki et al., 2015, 2017; Haere et al., 2016). PVA might be associated with lower lumbar T-score measured at 1 year after diagnosis, although neither the risk of osteoporosis at 5 years after diagnosis nor that of fractures at 2 years after diagnosis was higher in this group (Pekki et al., 2015, 2017). Decreased vitamin D and calcium levels might contribute to the impaired bone mineral density (Fang et al., 2017). Regarding other nutrients and minerals, zinc deficiency should be highlighted ( 39 vs. $14 \%$ in patients with PVA and MR, respectively; $p=0.0005$ ). The effect of PVA did not manifest itself regarding erythropoiesis: three studies reported similar hemoglobin levels and rate of anemia in patients with PVA and MR (Pekki et al., 2015; Fang et al., 2017; Mahadev et al., 2017). Rate of malignant tumors and 
TABLE 2 | Persistent villous atrophy in studies including only symptomatic or asymptomatic celiac patients.

\begin{tabular}{|c|c|c|c|c|c|c|}
\hline Study & $\begin{array}{l}\text { Symptoms assessed } \\
\text { prospectively? }\end{array}$ & $\begin{array}{l}\text { Symptoms assessed } \\
\text { at the time of } \\
\text { follow-up biopsy? }\end{array}$ & $\begin{array}{l}\text { Only strict } \\
\text { GFD? }\end{array}$ & Symptoms & $\begin{array}{l}\mathrm{N}^{0} \text { of pts with persistent } \\
\text { villous atrophy/total } \\
\text { (\% of total) }\end{array}$ & $\begin{array}{l}\text { Timing of follow-up } \\
\text { biopsy } \\
\text { (from CeD diagnosis) }\end{array}$ \\
\hline Bardella et al. (2007) & No & Yes & Yes & Not specified & $\begin{array}{l}71 \text { cases } / 114 \text { pts } \\
\text { (62) }\end{array}$ & $\begin{array}{l}2 \text { years (median) with 1-23 } \\
\text { years (range) }\end{array}$ \\
\hline Walters et al. (1995) & (?) & (?) & Yes & Not specified & $\begin{array}{l}9 \text { cases } / 17 \text { pts } \\
(53)\end{array}$ & 2 years (min) \\
\hline \multirow[t]{2}{*}{ Koskinen et al. (2010) } & Yes & Yes & (?) & Specified $^{a}$ & 21 cases/71 pts $(30)$ & 1 years \\
\hline & Yes & Yes & (?) & & $\begin{array}{l}2 \text { cases/105 pts } \\
\text { (2) }\end{array}$ & $\begin{array}{l}8 \text { years (median) with } 2-41 \\
\text { years (range) }\end{array}$ \\
\hline Cornell et al. (2016) & Yes & Yes & (?) & Not specified & $\begin{array}{l}9 \text { cases/20 pts } \\
(45)\end{array}$ & 4 m-36 years (range) \\
\hline \multicolumn{7}{|c|}{ SYMPTOMATIC POPULATION } \\
\hline
\end{tabular}

asymptoms included abdominal complaints, malabsorption, and extraintestinal symptoms.

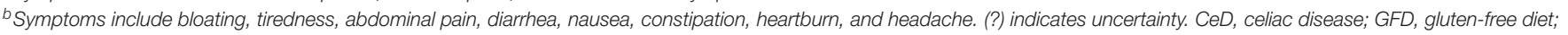
m, month; min, minimum; pts, patients.

that of lymphomas were not significantly different in histology groups in two studies 5 and 8 years after diagnosis (Pekki et al., 2015 , 2017). Similar neutral associations were found on the rate of cardiovascular diseases (Lebwohl et al., 2015a; Mahadev et al., 2017), serious infections including sepsis, streptococcal, pneumococcal, influenza, herpes zoster, and Clostridium difficile infections (Emilsson et al., 2018), and adverse pregnancy outcomes (Lebwohl et al., 2015b). MR seemed to be a protective factor against respiratory and dermatological diseases (Pekki et al., 2017). In contrast, anxiety and depression co-occurred more frequently with MR (Ludvigsson et al., 2018) as did epilepsy (Kurien et al., 2018). With respect to overall mortality, a higher rate was reported with PVA on 8-years follow-up (14 vs. 9\%) but the difference was not statistically significant $(p=0.259)$.

\section{Narrative Review}

In this subchapter, we summarize all available evidence published on the effect of MR and PVA on the outcomes of CeD.

\section{Symptoms, Symptom Scores and Quality of Life}

In asymptomatic patients, PVA rates ranged from 2 to $62 \%$ (Walters et al., 1995; Bardella et al., 2007; Koskinen et al., 2010; Cornell et al., 2016). In symptomatic patients, PVA rate was $38 \%$ (Mahadev et al., 2017) (Table 2). Six studies reported symptoms as dichotomies (symptomatic vs. asymptomatic) (Thornquist et al., 1993; Selby et al., 1999; Kaukinen et al., 2007; Carroccio et al., 2008; Fang et al., 2017; Leonard et al., 2017). In univariate analysis, the percentage points of symptomatic patients with PVA and MR were 55 vs. $70 \%(p=\mathrm{NS}$ ) (Leonard et al., 2017), 69 vs. $57 \%(p=0.076)$ (Fang et al., 2017), and 80 vs. 25\% ( $p<0.0001$ ) (Carroccio et al., 2008), respectively (Table 3). Only one study adjusted for reasonable co-variates where the adjusted OR was 3.2, 95\% CI: 1.6-6.4 $(p=0.001)$, indicating that patients with PVA tended to be symptomatic more often (Carroccio et al., 2008). Regarding the individual symptoms, only one study adjusted for co-variates where none of the symptoms in focus, that is, diarrhea, bloating, abdominal pain, nausea, tiredness, constipation, heartburn and headache, was significantly associated with PVA (Mahadev et al., 2017) (Table 3).

Five studies (Kaukinen et al., 2007; Tuire et al., 2012; Pekki et al., 2015, 2017; Haere et al., 2016) reported gastrointestinal symptom scores measured with the Gastrointestinal Symptom Rating Scale (GSRS) or its irritable bowel syndrome-adapted version (GSRS-IBS). Four of which performed statistical analysis and reported no association between histology and the scores consistently (Kaukinen et al., 2007; Pekki et al., 2015, 2017; Haere et al., 2016) (Table 4). Results were similar in quality of life measured with the Psychological General Well-Being Index (PGWB) and/or the 36-Item Short Form Health Survey (SF-36) (Kaukinen et al., 2007; Tuire et al., 2012; Pekki et al., 2015, 2017) (Table 4).

\section{Metabolic Bone Disease and Bone Fractures}

Four studies assessed bone mineral density in univariate analysis (Valdimarsson et al., 1994; Walters et al., 1995; Kaukinen et al., 2007; Pekki et al., 2015) (Table 5). Patients with PVA tended to have lower forearm, femoral and trochanter Z-scores (Valdimarsson et al., 1994); and lower femoral T-score with similar femoral Z- and lumbar T- and Z-scores than those with MR (Pekki et al., 2015). One study showed a reduced bone mineral density with PVA (OR $=24.5, p<0.0275$ favoring MR) (Walters et al., 1995). Two studies investigated the risk of osteoporosis in the long-term and reported conflicting results: a case-control study reported an increased frequency of osteoporosis with PVA (Kaukinen et al., 2007) but a cohort study failed to confirm (Pekki et al., 2015).

Three studies (Valdimarsson et al., 1994; Lebwohl et al., 2014; Pekki et al., 2017) reported no association between fractures and 
TABLE 3 | Presence of symptoms in celiac patients with persistent villous atrophy and mucosal recovery.

\begin{tabular}{|c|c|c|c|c|c|c|c|c|}
\hline Study & $\begin{array}{l}\text { Symptoms assessed } \\
\text { prospectively? }\end{array}$ & $\begin{array}{l}\text { Symptoms assessed } \\
\text { at the time of } \\
\text { follow-up biopsy? }\end{array}$ & $\begin{array}{l}\text { Only strict } \\
\text { GFD? }\end{array}$ & Symptoms & $\begin{array}{l}\mathrm{N}^{0} \text { of symptomatic } \\
\text { pts/total with } \\
\text { persistent villous } \\
\text { atrophy (\% of total) }\end{array}$ & $\begin{array}{l}\mathrm{N}^{0} \text { of symptomatic } \\
\text { pts/total with } \\
\text { mucosal recovery } \\
\text { (\% of total) }\end{array}$ & $\begin{array}{l}\text { Statistics } \\
\text { (atrophy vs. recovery) }\end{array}$ & $\begin{array}{l}\text { Timing of follow-up } \\
\text { biopsy } \\
\text { (from CeD diagnosis) }\end{array}$ \\
\hline
\end{tabular}

\begin{tabular}{|c|c|c|c|c|c|c|c|c|}
\hline \multicolumn{9}{|c|}{ SYMPTOMATIC vs. ASYMPTOMATIC PTS } \\
\hline $\begin{array}{l}\text { Carroccio } \\
\text { et al. (2008) }\end{array}$ & Yes & Yes & Yes & Specified ${ }^{a}$ & $\begin{array}{l}36 \text { cases } / 45 \text { pts } \\
(80)\end{array}$ & $\begin{array}{l}6 \text { cases } / 24 \text { pts } \\
\text { (25) }\end{array}$ & 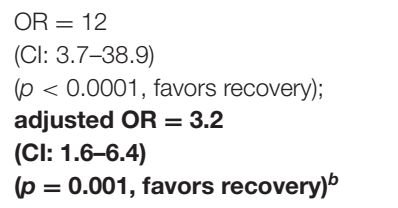 & $\begin{array}{l}6.5 y \text { and } 6.1 \text { y (means for } \\
\text { groups) with } 3.6 \text { y and } 4.6 y \\
\text { (SDs for groups) }\end{array}$ \\
\hline $\begin{array}{l}\text { Fang et al. } \\
\text { (2017) }\end{array}$ & No & Yes & Yes & Specified ${ }^{\mathrm{C}}$ & $\begin{array}{l}53 \text { cases } / 77 \mathrm{pts} \\
\text { (69) }\end{array}$ & $\begin{array}{l}116 \text { cases/203 pts } \\
(57)\end{array}$ & $\begin{array}{l}\mathrm{OR}=1.656 \\
(\mathrm{Cl}: 0.949-2.889)(p=0.076 \text {, favors } \\
\text { recovery) }\end{array}$ & 2 y (min) \\
\hline $\begin{array}{l}\text { Kaukinen } \\
\text { et al. (2007) }\end{array}$ & Yes & Nod & Yes & Not specified & $\begin{array}{l}4 \text { cases } / 13 \text { pts } \\
\text { (31) }\end{array}$ & Not reported & Not reported & $\begin{array}{l}8 \mathrm{y} \text { and } 10 \text { y (medians for } \\
\text { groups) with } 3-30 \text { y (range) }\end{array}$ \\
\hline $\begin{array}{l}\text { Leonard et al. } \\
\text { (2017) }\end{array}$ & No & Yes & No & Not specified & $\begin{array}{l}11 \text { cases/20 pts } \\
\text { (55) }\end{array}$ & $\begin{array}{l}58 \text { cases/83 pts } \\
(70)\end{array}$ & $p=N S$ & $\begin{array}{l}2.4 \mathrm{y} \text { (median) with } 1-12 \mathrm{y} \\
\text { (range) and 1.4-4 y }\left(\mathrm{Q}_{1}-\mathrm{Q}_{3}\right)\end{array}$ \\
\hline $\begin{array}{l}\text { Selby et al. } \\
\text { (1999) }\end{array}$ & (?) & $\mathrm{No}^{\mathrm{e}}$ & Yes & Not specified & $\begin{array}{l}5 \text { cases } / 26 \text { pts } \\
\text { (19) }\end{array}$ & $\begin{array}{l}3 \text { cases } / 24 \text { pts } \\
\text { (13) }\end{array}$ & Not reported & Not reported \\
\hline $\begin{array}{l}\text { Thornquist } \\
\text { et al. (1993) }\end{array}$ & Yes & $\mathrm{No}^{\mathrm{e}}$ & Yes & Specified ${ }^{f}$ & $\begin{array}{l}1 \text { case } / 4 \text { pts } \\
\text { (25) }\end{array}$ & $\begin{array}{l}7 \text { cases } / 9 \mathrm{pts} \\
\text { (78) }\end{array}$ & Not reported & $\begin{array}{l}17 \mathrm{y} \text { (mean) with } 15-18 \mathrm{y} \\
\text { (range?) }\end{array}$ \\
\hline \multicolumn{9}{|c|}{ SPECIFIC SYMPTOMs (PRESENT vs. ABSENT) } \\
\hline \multirow[t]{3}{*}{$\begin{array}{l}\text { Cammarota } \\
\text { et al. (2007) }\end{array}$} & Yes & Yes & No & Diarrhea & $\begin{array}{l}10 \text { cases } / 49 \text { pts } \\
(20)\end{array}$ & $\begin{array}{l}2 \text { cases } / 16 \text { pts } \\
\text { (13) }\end{array}$ & Not reported & $6-14 \mathrm{~m}$ (range) \\
\hline & & & & Bloating & $\begin{array}{l}9 \text { cases } / 49 \text { pts } \\
\text { (18) }\end{array}$ & $\begin{array}{l}3 \text { cases } / 16 \text { pts } \\
\text { (19) }\end{array}$ & & \\
\hline & & & & Abdominal pain & $\begin{array}{l}5 \text { cases } / 49 \text { pts } \\
\text { (10) }\end{array}$ & $\begin{array}{l}7 \text { cases } / 16 \text { pts } \\
(44)\end{array}$ & & \\
\hline \multirow[t]{3}{*}{$\begin{array}{l}\text { Carroccio } \\
\text { et al. (2008) }\end{array}$} & Yes & Yes & Yes & Typical symptoms & $\begin{array}{l}6 \text { cases } / 45 \mathrm{pts} \\
\text { (13) }\end{array}$ & $\begin{array}{l}0 \text { cases } / 24 \text { pts } \\
\text { (0) }\end{array}$ & $p=0.005$ (favors recovery) & $\begin{array}{l}6.5 \mathrm{y} \text { and } 6.1 \mathrm{y} \text { (means for } \\
\text { groups) with } 3.6 \mathrm{y} \text { and } 4.6 \mathrm{y} \\
\text { (SDs for groups) }\end{array}$ \\
\hline & & & & GERD-like symptoms & $\begin{array}{l}9 \text { cases } / 45 \text { pts } \\
(20)\end{array}$ & 3 cases/24 (13) & $p=0.01$ (favors recovery) & \\
\hline & & & & $\begin{array}{l}\text { lower abdominal } \\
\text { symptoms }\end{array}$ & $\begin{array}{l}21 \text { cases/45 pts } \\
\text { (47) }\end{array}$ & $\begin{array}{l}3 \text { cases/21 pts } \\
(14)\end{array}$ & $p=0.001$ (favors recovery) & \\
\hline \multirow[t]{3}{*}{$\begin{array}{l}\text { Ghazzawi } \\
\text { et al. (2014) }\end{array}$} & No & Yes & Yes & Abdominal pain & $\begin{array}{l}2 \text { cases } / 5 \text { pts } \\
(40)\end{array}$ & $\begin{array}{l}17 \text { cases } / 34 \text { pts } \\
(50)\end{array}$ & Not reported & $\begin{array}{l}2 \text { y (mean) with } 22 \mathrm{~m} \text { (SD) } \\
\text { and } 4-120 \mathrm{~m} \text { (range) }\end{array}$ \\
\hline & & & & Diarrhea & $\begin{array}{l}2 \text { cases } / 5 \mathrm{pts} \\
(40)\end{array}$ & $\begin{array}{l}5 \text { cases/34 pts } \\
\text { (15) }\end{array}$ & & \\
\hline & & & & Constipation & $\begin{array}{l}0 \text { cases } / 5 \mathrm{pts} \\
\text { (0) }\end{array}$ & $\begin{array}{l}5 \text { cases } / 34 \text { pts } \\
\text { (15) }\end{array}$ & & \\
\hline
\end{tabular}


TABLE 3 | Continued

\begin{tabular}{|c|c|c|c|c|c|c|c|c|}
\hline Study & $\begin{array}{l}\text { Symptoms assessed } \\
\text { prospectively? }\end{array}$ & $\begin{array}{l}\text { Symptoms assessed } \\
\text { at the time of } \\
\text { follow-up biopsy? }\end{array}$ & $\begin{array}{l}\text { Only strict } \\
\text { GFD? }\end{array}$ & Symptoms & $\begin{array}{l}\mathrm{N}^{0} \text { of symptomatic } \\
\text { pts/total with } \\
\text { persistent villous } \\
\text { atrophy (\% of total) }\end{array}$ & $\begin{array}{l}\mathrm{N}^{0} \text { of symptomatic } \\
\text { pts/total with } \\
\text { mucosal recovery } \\
\text { (\% of total) }\end{array}$ & $\begin{array}{l}\text { Statistics } \\
\text { (atrophy vs. recovery) }\end{array}$ & $\begin{array}{l}\text { Timing of follow-up } \\
\text { biopsy } \\
\text { (from CeD diagnosis) }\end{array}$ \\
\hline \multirow[t]{8}{*}{$\begin{array}{l}\text { Mahadev } \\
\text { et al. (2017) }\end{array}$} & \multirow[t]{8}{*}{ No } & \multirow[t]{8}{*}{ Yes } & \multirow[t]{8}{*}{ Yes } & Bloating & $\begin{array}{l}431 \text { cases/511 pts } \\
(84)\end{array}$ & $\begin{array}{l}736 \text { cases/834 pts } \\
\text { (88) }\end{array}$ & \multirow{8}{*}{$\begin{array}{l}p=0.04 \text { (favors atrophy), } p=0.001 \\
\text { (favors atrophy), } p=0.0009 \text { (favors } \\
\text { atrophy), and } p=0.04 \text { (favors } \\
\text { recovery) for bloating, abdominal } \\
\text { pain, nausea, and heartburn, } \\
\text { respectively; } p=\text { NS for the other } \\
\text { comparisons; } \\
\text { adjusted } p=\text { NS for all } \\
\text { comparisons }^{g}\end{array}$} & \multirow[t]{8}{*}{$4 \mathrm{y}$ (median) with $1 \mathrm{y}$ (min) } \\
\hline & & & & Abdominal pain & $\begin{array}{l}410 \text { cases/511 pts } \\
(80)\end{array}$ & $\begin{array}{l}724 \text { cases/834 pts } \\
\text { (87) }\end{array}$ & & \\
\hline & & & & Tiredness & $\begin{array}{l}421 \text { cases/511 pts } \\
\text { (82) }\end{array}$ & $\begin{array}{l}708 \text { cases } / 834 \\
\text { (85) }\end{array}$ & & \\
\hline & & & & Diarrhea & $\begin{array}{l}375 \text { cases/511 pts } \\
\text { (73) }\end{array}$ & $\begin{array}{l}643 \text { cases/834pts } \\
\text { (77) }\end{array}$ & & \\
\hline & & & & Nausea & $\begin{array}{l}239 \text { cases/511 pts } \\
(47)\end{array}$ & $\begin{array}{l}451 \text { cases/834 pts } \\
(54)\end{array}$ & & \\
\hline & & & & Constipation & $\begin{array}{l}263 \text { cases/511 pts } \\
(51)\end{array}$ & $\begin{array}{l}421 \text { cases/834 pts } \\
(50)\end{array}$ & & \\
\hline & & & & Heartburn & $\begin{array}{l}140 \text { cases/511 pts } \\
(27)\end{array}$ & $\begin{array}{l}186 \text { cases/834 pts } \\
(22)\end{array}$ & & \\
\hline & & & & Headache & $\begin{array}{l}112 \text { cases/511 pts } \\
\text { (22) }\end{array}$ & $\begin{array}{l}208 \text { cases/834 pts } \\
(25)\end{array}$ & & \\
\hline
\end{tabular}

Multivariate analyses are highlighted with bold. ${ }^{a}$ Symptoms included typical (chronic diarrhea, weight loss, anemia), GERD-like, and lower abdominal symptoms (abdominal pain, constipation). ${ }^{b}$ Analysis was adjusted for age, gender, duration of GFD, diagnostic histological severity. ' Symptoms included abdominal pain, bloating, nausea, fatigue, diarrhea, bloody stool, steatorrhea, and weight loss. ${ }^{d}$ Symptoms were assessed 4-5 years after the follow-up biopsy. eSymptoms were evaluated after follow-up biopsy (interval undetermined). ${ }^{f}$ Symptoms included gastrointestinal and malabsorptive symptoms, such as diarrhea, borborygmi, abdominal pain, fatty stool, and anemia. 9 The analysis was adjusted for age, gender, body mass index, duration of GFD, medications, and laboratory tests. (?) indicates uncertainty. CeD, celiac disease; Cl, confidence interval; GERD, gastroesophageal reflux disease; GFD, gluten-free diet; m, month; min, minimum; OR, odds ratio; pts, patients; NS, non-significant; $S D$, standard deviations; $Q_{1}-Q_{3}, 25$ and $75 \%$ quartiles; $y$, year. 
TABLE 4 | Symptom scores and quality of life indices in celiac patients with persistent villous atrophy and mucosal recovery.

\begin{tabular}{|c|c|c|c|c|c|c|c|c|c|c|}
\hline \multirow[t]{2}{*}{ Study } & \multirow{2}{*}{$\begin{array}{l}\text { Symptoms } \\
\text { assessed } \\
\text { prospectively? }\end{array}$} & \multirow{2}{*}{$\begin{array}{l}\text { Symptoms assessed } \\
\text { at the time of } \\
\text { follow-up biopsy? }\end{array}$} & \multirow{2}{*}{$\begin{array}{l}\text { Only strict } \\
\text { GFD? }\end{array}$} & \multirow[t]{2}{*}{ Tool } & \multicolumn{2}{|c|}{ Persistent villous atrophy } & \multicolumn{2}{|c|}{ Mucosal recovery } & \multirow{2}{*}{$\begin{array}{l}\text { Statistics } \\
\text { (atrophy vs. } \\
\text { recovery) }\end{array}$} & \multirow{2}{*}{$\begin{array}{l}\text { Timing of follow-up } \\
\text { biopsy (from CeD } \\
\text { diagnosis) }\end{array}$} \\
\hline & & & & & $\begin{array}{l}\mathrm{N}^{0} \text { of } \\
\text { pts }\end{array}$ & Score & $\begin{array}{l}\mathrm{N}^{0} \text { of } \\
\text { pts }\end{array}$ & Score & & \\
\hline \multicolumn{11}{|c|}{ GASTROINTESTINAL SYMPTOM SCORES } \\
\hline Pekki et al. (2017) & Yes & $\mathrm{No}^{\mathrm{a}}$ & No & GSRS & 200 & Not reported & 276 & Not reported & $p=\mathrm{NS}$ & $1 \mathrm{y}$ \\
\hline $\begin{array}{l}\text { Kaukinen et al. } \\
(2007)\end{array}$ & Yes & Yes & Yes & GSRS & 13 & $\begin{array}{l}\text { mean: } 2.0(\mathrm{Cl}: \\
1.4-2.6)\end{array}$ & 18 & $\begin{array}{l}\text { mean: } 2.0(\mathrm{Cl}: \\
1.7-2.3)\end{array}$ & $p=\mathrm{NS}$ & $\begin{array}{l}8 \mathrm{y} \text { and } 10 \mathrm{y} \text { (medians for } \\
\text { groups) with } 3-30 \text { y (range) }\end{array}$ \\
\hline \multirow[t]{2}{*}{ Pekki et al. (2015) } & Yes & Yes & No & GSRS & 85 & $\begin{array}{l}\text { Median: } 1.5 \\
\left(Q_{1}-Q_{3}: 1.3-1.8\right)\end{array}$ & 178 & $\begin{array}{l}\text { Median: } 1.5 \\
\left(Q_{1}-Q_{3}: 1.3-2.0\right)\end{array}$ & $p=0.321$ & $1 \mathrm{y}$ \\
\hline & & $\mathrm{No}^{\mathrm{a}}$ & No & GSRS & $\sum 44^{b}$ & $\begin{array}{l}\text { Median: } 1.6 \\
\left(Q_{1}-Q_{3}: 1.0-4.0\right)\end{array}$ & $\sum 44^{b}$ & $\begin{array}{l}\text { Median: } 1.9 \\
\left(Q_{1}-Q_{3}: 1.0-3.0\right)\end{array}$ & $p=0.132$ & 5 y (median) \\
\hline Tuire et al. (2012) & Yes & Yes & Yes & GSRS $^{c}$ & 7 & $\begin{array}{l}\text { Mean: } 1.73(\mathrm{Cl}: \\
1.39-2.07)\end{array}$ & 170 & Not reported & Not reported & $2-41$ y (range) \\
\hline Haere et al. (2016) & Yes & Yes & No & GSRS-IBS $^{d}$ & 7 & $\begin{array}{l}\text { Mean: } 25.1 \text { (SD } \\
\text { 9.9) }\end{array}$ & 116 & $\begin{array}{l}\text { Mean: } 26.4 \text { (SD: } \\
\text { 9.9) }\end{array}$ & $p=\mathrm{NS}$ & $\begin{array}{l}9.3 \mathrm{y} \text { (mean) with } 5 \mathrm{y}(S D) \\
\text { and } 2 \mathrm{y} \text { (min) }\end{array}$ \\
\hline \multicolumn{11}{|c|}{ QUALITY OF LIFE SCORES } \\
\hline \multirow[t]{2}{*}{ Pekki et al. (2017) } & Yes & $\mathrm{No}^{\mathrm{a}}$ & No & PGWB & 200 & Not reported & 276 & Not reported & $p=\mathrm{NS}$ & $1 y$ \\
\hline & & & & SF-36 & & & & & $p=\mathrm{NS}$ & \\
\hline $\begin{array}{l}\text { Kaukinen et al. } \\
\text { (2007) }\end{array}$ & Yes & Yes & Yes & PGWB & 13 & $\begin{array}{l}\text { Mean: } 101 \text { (Cl: } \\
\text { 94-108) }\end{array}$ & 18 & $\begin{array}{l}\text { Mean: } 101 \text { (Cl: } \\
\text { 92-110) }\end{array}$ & $p=N S$ & $\begin{array}{l}8 \mathrm{y} \text { and } 10 \mathrm{y} \text { (medians for } \\
\text { groups) with } 3-30 \text { y (range) }\end{array}$ \\
\hline \multirow[t]{2}{*}{ Pekki et al. (2015) } & Yes & Yes & No & PGWB & 85 & $\begin{array}{l}\text { Median: } 112 \\
\left(Q_{1}-Q_{3}: 102-118\right)\end{array}$ & 178 & $\begin{array}{l}\text { Median: } 111 \\
\left(Q_{1}-Q_{3}: 100-117\right)\end{array}$ & $p=0.699$ & $1 \mathrm{y}$ \\
\hline & & $\mathrm{No}^{\mathrm{a}}$ & No & PGWB & $\sum 44^{b}$ & $\begin{array}{l}\text { median: } 109 \\
\left(Q_{1}-Q_{3}: 52-124\right)\end{array}$ & $\sum 44^{b}$ & $\begin{array}{l}\text { Median: } 108 \\
\left(Q_{1}-Q_{3}: 62-121\right)\end{array}$ & $p=0.416$ & 5 y (median) \\
\hline Tuire et al. (2012) & Yes & Yes & Yes & PGWB & 7 & $\begin{array}{l}\text { Mean: } 112.7 \text { (Cl: } \\
110.6-114.8)\end{array}$ & 170 & Not reported & Not reported & 2-41 y (range) \\
\hline
\end{tabular}

${ }^{a}$ Scores were assessed years after the follow-up biopsy. ${ }^{b}$ The number of patients were not reported for atrophic and recovery groups separately. ${ }^{c}$ Subdimensions of GSRS were reported within the original article in detail. ${ }^{d} A 3$-day modified version of the original tool was used. CeD, celiac disease; Cl, confidence interval; GFD, gluten-free diet; GSRS, Gastrointestinal Symptom Rating Scale; GSRS-IBS: Gastrointestinal Symptom Rating Scale for Irritable Bowel Syndrome; min, minimum; NS, non-significant: PGWB, Psychological General Well-Being: pts, patients; SF-36, Short Form Health Survey-36; Q1-Q3, 25\% and 75\% quartiles; SD: standard deviation. 
TABLE 5 | Bone mineral density in celiac patients with persistent villous atrophy and mucosal recovery

\begin{tabular}{|c|c|c|c|c|c|c|c|c|c|c|}
\hline \multirow[t]{2}{*}{ Study } & \multirow{2}{*}{$\begin{array}{l}\text { Parameter of } \\
\text { interest } \\
\text { assessed } \\
\text { prospectively? }\end{array}$} & \multirow{2}{*}{$\begin{array}{l}\text { Parameter of } \\
\text { interest } \\
\text { assessed at the } \\
\text { time of follow-up } \\
\text { biopsy? }\end{array}$} & \multirow{2}{*}{$\begin{array}{l}\text { Only strict } \\
\text { GFD? }\end{array}$} & \multirow[t]{2}{*}{ Bone mineral density } & \multicolumn{2}{|c|}{ Persistent villous atrophy } & \multicolumn{2}{|c|}{ Mucosal recovery } & \multirow{2}{*}{$\begin{array}{l}\text { Statistics } \\
\text { (atrophy vs. } \\
\text { recovery) }\end{array}$} & \multirow{2}{*}{$\begin{array}{l}\text { Timing of follow-up } \\
\text { biopsy } \\
\text { (from CeD diagnosis }\end{array}$} \\
\hline & & & & & Scores & $\begin{array}{l}\mathrm{N}^{0} \text { of } \\
\text { pts }\end{array}$ & Scores & $\begin{array}{l}\mathbf{N}^{0} \text { of } \\
\text { pts }\end{array}$ & & \\
\hline \multicolumn{11}{|l|}{ T- AND Z-SCORES } \\
\hline \multirow[t]{4}{*}{ Pekki et al. (2015) } & Yes & Yes & No & Lumbar (T-score) & $\begin{array}{l}\text { Median: }-1.1 \\
\left(Q_{1}-Q_{3}:-2.2 \text { to-0.2) }\right.\end{array}$ & $\sum 152^{\mathrm{a}}$ & $\begin{array}{l}\text { Median: }-0.7 \\
\left(Q_{1}-Q_{3}:-1.7 \text { to } 0.4\right)\end{array}$ & $\sum 152^{\mathrm{a}}$ & $p=0.117$ & $1 \mathrm{y}$ \\
\hline & & & & Femoral neck (T-score) & $\begin{array}{l}\text { Median: }-1.1 \\
\left(Q_{1}-Q_{3}:-1.9 \text { to }-0.5\right)\end{array}$ & $85 \mathrm{pts}$ & $\begin{array}{l}\text { Median: }-0.6 \\
\left(Q_{1}-Q_{3}:-1.5 \text { to } 0.0\right)\end{array}$ & $178 \mathrm{pts}$ & $\begin{array}{l}p=0.024 \\
\text { (favors recovery) }\end{array}$ & \\
\hline & & & & Lumbar (Z-score) & $\begin{array}{l}\text { Median: }-0.5 \\
\left(Q_{1}-Q_{3}:-1.3 \text { to }-0.1\right)\end{array}$ & $\sum 159^{a}$ & $\begin{array}{l}\text { Median: } 0.1 \\
\left(Q_{1}-Q_{3}:-1.1 \text { to } 0.9\right)\end{array}$ & $\sum 159^{a}$ & $p=0.376$ & \\
\hline & & & & Femoral neck (Z-score) & $\begin{array}{l}\text { Median: }-0.5 \\
\left(Q_{1}-Q_{3}:-0.7 \text { to }-0.1\right)\end{array}$ & $\sum 140^{a}$ & $\begin{array}{l}\text { Median: }-0.3 \\
\left(Q_{1}-Q_{3}:-0.6 \text { to } 0.3\right)\end{array}$ & $\sum 140^{a}$ & $p=0.323$ & \\
\hline \multirow[t]{4}{*}{$\begin{array}{l}\text { Valdimarsson et al. } \\
\text { (1994) }\end{array}$} & Yes & (?) & No & Forearm (Z-score) & Not reported & 13 pts & Not reported & $17 \mathrm{pts}$ & $\begin{array}{l}P<0.01 \\
\text { (favors recovery) }\end{array}$ & $\begin{array}{l}8 \mathrm{y} \text { and } 9 \mathrm{y} \text { (medians } \\
\text { for groups) with } 4-14 \mathrm{y} \\
\text { (range) }\end{array}$ \\
\hline & & & & Femoral neck (Z-score) & & & & & $\begin{array}{l}p<0.01 \\
\text { (favors recovery) }\end{array}$ & \\
\hline & & & & $\begin{array}{l}\text { Femoral trochanter } \\
\text { (Z-score) }\end{array}$ & & & & & $\begin{array}{l}p<0.05 \\
\text { (favors recovery) }\end{array}$ & \\
\hline & & & & & $\begin{array}{l}\text { Affected pts/total } \\
\text { persistent villous atrc } \\
\text { of total) }\end{array}$ & $\begin{array}{l}\text { Ith } \\
\text { ophy (\% }\end{array}$ & $\begin{array}{l}\text { Affected pts/tot } \\
\text { mucosal recovery }\end{array}$ & $\begin{array}{l}\text { I with } \\
\text { of total) }\end{array}$ & & \\
\hline \multicolumn{11}{|c|}{ OSTEOPOROSIS AND OSTEOPENIA } \\
\hline \multirow[t]{2}{*}{$\begin{array}{l}\text { Kaukinen et al. } \\
(2007)\end{array}$} & Yes & Yes & Yes & $\begin{array}{l}\text { Osteoporosis (T-score } \\
\leq-2.5 \mathrm{SD})\end{array}$ & $\begin{array}{c}7 \text { cases/12 pts } \\
\text { (58) }\end{array}$ & & $\begin{array}{c}4 \text { cases } / 18 p \\
\text { (22) }\end{array}$ & & $p=0.04$ & $\begin{array}{l}8 \mathrm{y} \text { and } 10 \text { y (medians } \\
\text { for groups) with } 3-30 \mathrm{y} \\
\text { (range) }\end{array}$ \\
\hline & & & & $\begin{array}{l}\text { Osteopenia (T-score } \\
\text { between }-1.0 \text { and } \\
-2.4 S D \text { ) }\end{array}$ & $\begin{array}{c}4 \text { cases/12 pts } \\
\text { (33) }\end{array}$ & & 8 cases $/ 18 \mathrm{pt}$ & (44) & $p=\mathrm{NS}$ & \\
\hline Pekki et al. (2015) & Yes & Nob & No & $\begin{array}{l}\text { Osteoporosis (T- and } \\
\text { Z-scores) }\end{array}$ & $\begin{array}{c}8 \text { cases/71 pts } \\
\text { (14) }\end{array}$ & & $\begin{array}{c}16 \text { cases/ } 134 \\
(13)\end{array}$ & & $p=0.850$ & 5 y (median) \\
\hline $\begin{array}{l}\text { Walters et al. } \\
\text { (1995) }\end{array}$ & Yes & Nob & Yes & Osteopenia (Z-score) & $\begin{array}{c}7 \text { cases } / 9 \text { pts } \\
(78)\end{array}$ & & $\begin{array}{c}1 \text { case } / 8 \mathrm{pt} \\
(13)\end{array}$ & & $\begin{array}{l}p<0.0275 \\
\text { (favors recovery) }\end{array}$ & 2 y (min) \\
\hline
\end{tabular}

All measurements were performed with dual-energy X-ray absorptiometry except in forearm bone mineral density in the study of Valdimarsson et al. (1994) which used single-photon absorptiometry. ${ }^{2}$ The number of patients were not reported for atrophic and recovery groups separately. ${ }^{b}$ Parameter was assessed years after follow-up biopsy. (?) indicates uncertainty. GFD, gluten-free diet; $m$, month; min, minimum; NS, non-significant; pts, patients $Q_{1}-Q_{3}, 25 \%$ and $75 \%$ quartiles; SD, standard deviation; y, year. 
TABLE 6 | Fractures in celiac patients with persistent villous atrophy and mucosal recovery.

\begin{tabular}{|c|c|c|c|c|c|c|c|c|}
\hline Study & $\begin{array}{l}\text { Events } \\
\text { assessed } \\
\text { prospectively? }\end{array}$ & $\begin{array}{l}\text { Only } \\
\text { strict } \\
\text { GFD? }\end{array}$ & $\begin{array}{l}\text { Type of } \\
\text { fracture }\end{array}$ & $\begin{array}{l}\text { Persistent } \\
\text { villous atrophy }\end{array}$ & $\begin{array}{l}\text { Mucosal } \\
\text { recovery }\end{array}$ & $\begin{array}{l}\text { Statistics } \\
\text { (atrophy vs. } \\
\text { recovery) }\end{array}$ & $\begin{array}{l}\text { Timing of } \\
\text { follow-up biopsy } \\
\text { (from CeD } \\
\text { diagnosis) }\end{array}$ & Follow-up period \\
\hline $\begin{array}{l}\text { Valdimarsson } \\
\text { et al. (1994) }\end{array}$ & Yes & No & Any fracture & $\begin{array}{l}9 \text { fractures in } 6 \\
\text { cases/13 pts }\end{array}$ & $\begin{array}{l}5 \text { fractures in } 5 \\
\text { cases/17 pts }\end{array}$ & $\begin{array}{l}p=N S \text { for the } \\
\text { number of patients } \\
\text { with fractures }\end{array}$ & $\begin{array}{l}8 \mathrm{y} \text { and } 9 \mathrm{y} \\
\text { (medians for } \\
\text { groups) with 4-14 } \\
\text { y (range) }\end{array}$ & Not reported \\
\hline \multirow[t]{2}{*}{$\begin{array}{l}\text { Lebwohl et al. } \\
\text { (2014) }\end{array}$} & No & (?) & Any fracture ${ }^{a}$ & $\begin{array}{l}492 \text { cases/34409 } \\
\text { PYO }\end{array}$ & $\begin{array}{l}483 \text { cases } / 36418 \\
\text { PYO }\end{array}$ & $\begin{array}{l}\mathrm{HR}=0.93 \\
(\mathrm{Cl}: 0.82-1.06)^{b}\end{array}$ & $6 \mathrm{~m}-5$ y (range) & \multirow{2}{*}{$\begin{array}{l}\text { 8.6 y (median) with } \\
6.9-15.7 \text { y }\left(Q_{1}-Q_{3}\right) \\
\text { from follow-up } \\
\text { biopsy }\end{array}$} \\
\hline & & & $\begin{array}{l}\text { Likely } \\
\text { osteoporotic } \\
\text { fracture }^{\mathrm{a}}\end{array}$ & $\begin{array}{l}124 \text { cases/20617 } \\
\text { PYO }\end{array}$ & $\begin{array}{l}96 \text { cases } / 11916 \\
\text { PYO }\end{array}$ & $\begin{array}{l}\mathrm{HR}=1.11 \\
(\mathrm{Cl}: 0.84-1.46)^{\mathrm{b}}\end{array}$ & & \\
\hline
\end{tabular}

Multivariate analyses are highlighted with bold. ${ }^{a}$ Detailed data are available in the original article. ${ }^{b}$ The analysis was adjusted for age, gender, duration of celiac disease, calendar period, and educational attainment. (?) indicates uncertainty. Cl, confidence interval; GFD, gluten-free diet; HR, hazard ratio (reference group: mucosal recovery); NS, non-significant; PYO, person-years observation; $Q_{1}-Q_{3}: 25$ and $75 \%$ quartiles.

follow-up histology, except in the frequency of hip fractures being seemingly increased after a 5-years follow-up with PVA (adjusted $\mathrm{HR}=2.18$, CI: 1.17-4.05) (Lebwohl et al., 2014) (Table 6).

\section{Micro- and Macronutrients}

Table 7 summarizes the laboratory findings.

The association of water-soluble vitamins and follow-up histology is understudied. We found the numerical values of vitamins $B_{2}$ and $B_{6}$ levels or their laboratory indicators in patients with MR and PVA but the studies did not perform statistical comparison (Dickey et al., 2008). Regarding vitamins $\mathrm{B}_{12}$ and $\mathrm{B}_{9}$ (folic acid) levels, studies found no difference between groups by histology (Pekki et al., 2015; Fang et al., 2017). No reports are available on other water-soluble vitamins.

Reports on fat-soluble vitamins are scarce. Only numerical data are available on vitamin A without statistical comparison made by the authors (Valdimarsson et al., 1994). Patients with PVA tended to have lower 25-hydroxyvitamin D level [and total calcium (Fang et al., 2017)], though serum parathyroid hormone (Valdimarsson et al., 1994; Pekki et al., 2015), ionized calcium (Valdimarsson et al., 1994; Pekki et al., 2015) and alkaline phosphatase (Valdimarsson et al., 1994) seemed similar in patients with MR and PVA.

Zinc but not copper deficiency may be associated with PVA ( 39 vs. $14 \%$ for zinc deficiency with PVA and MR, respectively, $p=0.0005$ ) (Fang et al., 2017).

\section{Anemia}

Nine studies reported on hemoglobin, four studies in dichotomies (aaemic vs. non-anemic) and five as continuous data (Thornquist et al., 1993; Kemppainen et al., 1998; Ciacci et al., 2002; Cammarota et al., 2007; Kaukinen et al., 2007; Tuire et al., 2012; Pekki et al., 2015; Fang et al., 2017; Mahadev et al., 2017), of which four reported statistical comparisons (Kaukinen et al., 2007; Pekki et al., 2015; Fang et al., 2017; Mahadev et al., 2017) (Table 8). Hemoglobin levels proved to be not statistically different in two studies (Kaukinen et al., 2007; Pekki et al., 2015). Another two studies examined the frequency of anemia (Fang et al., 2017; Mahadev et al., 2017), of which one found it more frequent with PVA (22 vs. 18\%, $p=0.04$ ) (Mahadev et al., 2017). However, the association was not confirmed after adjusting for co-variates $(p=0.236)$.

\section{Body Mass Index}

Of the six studies reporting body mass index, three provided statistical evidence on having no significant difference between groups while the other three reported numerical values without analysis (Valdimarsson et al., 1994; Kaukinen et al., 2007; Dickey et al., 2008; Tuire et al., 2012; Pekki et al., 2015; Cornell et al., 2016) (Table 9).

\section{Malignant Tumors and Other Co-morbid Conditions}

Four studies reported the overall rate of malignant tumors during follow-up (Kaukinen et al., 2007; Tuire et al., 2012; Pekki et al., $2015,2017)$, two of which performed univariate analysis where event rates were 4.5 vs. $5.1 \%(p=0.762)$ and 11.3 vs. $6 \%(p=$ $0.116)$ with PVA vs. MR, respectively (Pekki et al., 2015, 2017)

\section{(Table 10).}

Incidence of lymphomas was 1.4 vs. $1.6 \%(p=0.968)$ with PVA vs. MR in univariate analysis (Pekki et al., 2015). In a registry analysis, PVA had an adjusted HR of 2.26 (CI: 1.18-4.34) for the overall rate of lymphomas, being true for the subgroup of nonHodgkin lymphomas but not for the subset of T-cell lymphomas (Lebwohl et al., 2013b) (Table 10).

Sporadic records concerned other co-morbid conditions. Cardiovascular diseases including heart failure and atrial fibrillation (Lebwohl et al., 2015a), hypertension (Mahadev et al., 2017), serious infections (including sepsis, streptococcal, 
TABLE 7 | Vitamins, minerals, and homocysteine in celiac patients with persistent villous atrophy and mucosal recovery.

\begin{tabular}{|c|c|c|c|c|c|c|c|c|c|c|c|c|}
\hline \multirow[t]{2}{*}{ Study } & \multirow{2}{*}{$\begin{array}{l}\text { Parameter of } \\
\text { interest } \\
\text { assessed } \\
\text { prospectively? }\end{array}$} & \multirow{2}{*}{$\begin{array}{l}\text { Parameter of } \\
\text { interest } \\
\text { assessed at the } \\
\text { time of follow-up } \\
\text { biopsy? }\end{array}$} & \multirow{2}{*}{$\begin{array}{l}\text { Only } \\
\text { strict } \\
\text { GFD? }\end{array}$} & \multirow[t]{2}{*}{ Laboratory study } & \multicolumn{3}{|c|}{ Persistent villous atrophy } & \multicolumn{3}{|c|}{ Mucosal recovery } & \multirow{2}{*}{$\begin{array}{l}\text { Statistics } \\
\text { (atrophy vs. } \\
\text { recovery) }\end{array}$} & \multirow{2}{*}{$\begin{array}{l}\text { Timing of } \\
\text { follow-up } \\
\text { biopsy (from } \\
\text { CeD } \\
\text { diagnosis) }\end{array}$} \\
\hline & & & & & $\begin{array}{l}\text { Total } \mathbf{N}^{0} \text { of } \\
\text { pts }\end{array}$ & $\begin{array}{l}\mathrm{N}^{0} \text { of pts with } \\
\text { deficiency }(\% \text { of } \\
\text { total) }\end{array}$ & Level & $\begin{array}{l}\text { Total } N^{0} \text { of } \\
\text { pts }\end{array}$ & $\begin{array}{l}\mathrm{N}^{0} \text { of pts with } \\
\text { deficiency (\% of } \\
\text { total) }\end{array}$ & Level & & \\
\hline \multicolumn{13}{|c|}{ VITAMIN B ${ }_{2}$ (RIBOFLAVIN) } \\
\hline $\begin{array}{l}\text { Dickey et al. } \\
\text { (2008) }\end{array}$ & Yes & Yes & Yes & EGRAC & 24 & & $\begin{array}{l}\text { Mean: } 1.38 \\
(S D: 0.19)\end{array}$ & 41 & & $\begin{array}{l}\text { Mean: } 1.28 \\
\text { (SD: } 0.13)\end{array}$ & Not reported & 1 y (min) \\
\hline \multicolumn{13}{|c|}{ VITAMIN B 6 (PYRIDOXINE) } \\
\hline $\begin{array}{l}\text { Dickey et al. } \\
\text { (2008) }\end{array}$ & Yes & Yes & Yes & $\begin{array}{l}\text { Plasma pyridoxal } \\
\text { phosphate (nmol/l, } \\
\text { vitamin) }\end{array}$ & 24 & & $\begin{array}{l}\text { Mean: } 69.9 \\
(S D: 24.0)\end{array}$ & 41 & & $\begin{array}{l}\text { Mean: } 91.0 \\
\text { (SD: 43.3) }\end{array}$ & Not reported & 1 y (min) \\
\hline \multicolumn{13}{|c|}{ VITAMIN Bg (FOLIC ACID) } \\
\hline $\begin{array}{l}\text { Dickey et al. } \\
\text { (2008) }\end{array}$ & Yes & Yes & Yes & $\begin{array}{l}\text { Erythrocyte folic } \\
\text { acid (nmol/l) }\end{array}$ & 24 & & $\begin{array}{l}\text { Mean: } 900 \\
(S D: 574)\end{array}$ & 41 & & $\begin{array}{l}\text { Mean: } 1048 \\
\text { (SD: 791) }\end{array}$ & Not reported & 1 y (min) \\
\hline $\begin{array}{l}\text { Dickey et al. } \\
\text { (2008) }\end{array}$ & Yes & Yes & Yes & $\begin{array}{l}\text { Serum folic acid } \\
(\mathrm{nmol} / \mathrm{l})\end{array}$ & 24 & & $\begin{array}{l}\text { Mean: } 23.5 \\
\text { (SD: 22.8) }\end{array}$ & 41 & & $\begin{array}{l}\text { Mean: } 25.7 \\
\text { (SD: } 31.5)\end{array}$ & Not reported & 1 y (min) \\
\hline $\begin{array}{l}\text { Kemppainen } \\
\text { et al. (1998) }\end{array}$ & No & Yes & (?) & $\begin{array}{l}\text { Erythrocyte folic } \\
\text { acid (cut-off: not } \\
\text { reported) }\end{array}$ & 37 & $3(8)$ & & 3 & $0(0)$ & & Not reported & $1 \mathrm{y}$ \\
\hline $\begin{array}{l}\text { Pekki et al. } \\
\text { (2015) }\end{array}$ & Yes & Yes & No & $\begin{array}{l}\text { Erythrocyte folic } \\
\text { acid (nmol/l) }\end{array}$ & $\sum 142^{\mathrm{a}}$ & & $\begin{array}{l}\text { Median: } 548 \\
\left(Q_{1}-Q_{3}:\right. \\
424-676)\end{array}$ & $\sum 142^{a}$ & & $\begin{array}{l}\text { Median: } 508 \\
\left(Q_{1}-Q_{3}:\right. \\
383-650)\end{array}$ & $p=0.547$ & $1 \mathrm{y}$ \\
\hline $\begin{array}{l}\text { Thornquist } \\
\text { et al. (1993) }\end{array}$ & Yes & $\mathrm{No}^{\mathrm{b}}$ & No & Serum folic acid & 4 & & Not reported ${ }^{d}$ & 9 & & Not reported ${ }^{d}$ & Not reported & $\begin{array}{l}17 \text { y (mean) } \\
\text { with 15-18 y } \\
\text { (range?) }\end{array}$ \\
\hline $\begin{array}{l}\text { Tuire et al. } \\
\text { (2012) }\end{array}$ & Yes & Yes & Yes & $\begin{array}{l}\text { Erythrocyte folic } \\
\text { acid (nmol/l) }\end{array}$ & 7 & & $\begin{array}{l}\text { Median: } 486 \\
\text { (range: } \\
243-1,849 \text { ) }\end{array}$ & 170 & & Not reported & Not reported & $2-41$ y (range) \\
\hline \multicolumn{13}{|c|}{ VITAMIN B ${ }_{12}$ (COBALAMIN) } \\
\hline $\begin{array}{l}\text { Dickey et al. } \\
\text { (2008) }\end{array}$ & Yes & Yes & Yes & $\begin{array}{l}\text { Serum vitamin } B_{12} \\
(\mathrm{pmol} / \mathrm{l})\end{array}$ & 24 & & $\begin{array}{l}\text { Mean: } 242.5 \\
\text { (SD: 105.8) }\end{array}$ & 41 & & $\begin{array}{l}\text { Mean: } 276.6 \\
\text { (SD: 137.1) }\end{array}$ & Not reported & $1 y(\mathrm{~min})$ \\
\hline $\begin{array}{l}\text { Fang et al. } \\
\text { (2017) }\end{array}$ & No & $\mathrm{No}^{\mathrm{C}}$ & Yes & $\begin{array}{l}\text { Serum vitamin } B_{12} \\
\text { (cut-off: } 180 \mathrm{ng} / \mathrm{l})\end{array}$ & 60 & 2 (3) & & 175 & $3(2)$ & & $p=0.6023$ & $2 y(\min )$ \\
\hline $\begin{array}{l}\text { Kemppainen } \\
\text { et al. (1998) }\end{array}$ & No & Yes & (?) & $\begin{array}{l}\text { Serum vitamin } \mathrm{B}_{12} \\
\text { (cut-off: not } \\
\text { reported) }\end{array}$ & 37 & 1 (3) & & 3 & $0(0)$ & & Not reported & $1 \mathrm{y}$ \\
\hline $\begin{array}{l}\text { Pekki et al. } \\
\text { (2015) }\end{array}$ & Yes & Yes & No & $\begin{array}{l}\text { Serum vitamin } B_{12} \\
(\mathrm{pmol} / \mathrm{l})\end{array}$ & 85 & & $\begin{array}{l}\text { Median: } 383 \\
\left(Q_{1}-Q_{3}:\right. \\
286-464)\end{array}$ & 178 & & $\begin{array}{l}\text { Median: } 341 \\
\left(Q_{1}-Q_{3}:\right. \\
269-428)\end{array}$ & $p=0.153$ & $1 \mathrm{y}$ \\
\hline $\begin{array}{l}\text { Thornquist } \\
\text { et al. (1993) }\end{array}$ & Yes & $\mathrm{No}^{\mathrm{b}}$ & No & Serum vitamin $B_{12}$ & 4 & & Not reported ${ }^{d}$ & 9 & & Not reported ${ }^{d}$ & Not reported & $\begin{array}{l}17 \mathrm{y} \text { (mean) } \\
\text { with 15-18 y } \\
\text { (range ?) }\end{array}$ \\
\hline
\end{tabular}


TABLE 7 | Continued

\begin{tabular}{|c|c|c|c|c|c|c|c|c|c|c|c|c|}
\hline \multirow[t]{2}{*}{ Study } & \multirow{2}{*}{$\begin{array}{l}\text { Parameter of } \\
\text { interest } \\
\text { assessed } \\
\text { prospectively? }\end{array}$} & \multirow{2}{*}{$\begin{array}{l}\text { Parameter of } \\
\text { interest } \\
\text { assessed at the } \\
\text { time of follow-up } \\
\text { biopsy? }\end{array}$} & \multirow{2}{*}{$\begin{array}{l}\text { Only } \\
\text { strict } \\
\text { GFD? }\end{array}$} & \multirow[t]{2}{*}{ Laboratory study } & \multicolumn{3}{|c|}{ Persistent villous atrophy } & \multicolumn{3}{|c|}{ Mucosal recovery } & \multirow{2}{*}{$\begin{array}{l}\text { Statistics } \\
\text { (atrophy vs. } \\
\text { recovery) }\end{array}$} & \multirow{2}{*}{$\begin{array}{l}\text { Timing of } \\
\text { follow-up } \\
\text { biopsy (from } \\
\text { CeD } \\
\text { diagnosis) }\end{array}$} \\
\hline & & & & & $\begin{array}{l}\text { Total } \mathbf{N}^{0} \text { of } \\
\text { pts }\end{array}$ & $\begin{array}{l}\mathrm{N}^{0} \text { of pts with } \\
\text { deficiency (\% of } \\
\text { total) }\end{array}$ & Level & $\begin{array}{l}\text { Total } \mathbf{N}^{0} \text { of } \\
\text { pts }\end{array}$ & $\begin{array}{l}\mathrm{N}^{0} \text { of pts with } \\
\text { deficiency (\% of } \\
\text { total) }\end{array}$ & Level & & \\
\hline \multicolumn{13}{|c|}{ VITAMIN A (RETINOL) } \\
\hline $\begin{array}{l}\text { Kemppainen } \\
\text { et al. (1998) }\end{array}$ & No & Yes & (?) & $\begin{array}{l}\text { Serum vitamin A } \\
\text { (cut-off: not } \\
\text { reported) }\end{array}$ & 37 & $5(14)$ & & 3 & $0(0)$ & & Not reported & $1 \mathrm{y}$ \\
\hline \multicolumn{13}{|c|}{ VITAMIN D (CHOLECALCIFEROL) } \\
\hline $\begin{array}{l}\text { Fang et al. } \\
\text { (2017) }\end{array}$ & No & $\mathrm{No}^{\mathrm{C}}$ & Yes & $\begin{array}{l}\text { Serum total } \\
\text { 25-hydroxy } \\
\text { vitamin } D_{2} \text { and } D_{3} \\
\text { (cut-off: } 25 \mathrm{ng} / \mathrm{ml} \text { ) }\end{array}$ & 55 & $17(31)$ & & 174 & $14(8)$ & & $\begin{array}{l}p=0.0001 \\
\text { (favors } \\
\text { recovery) }\end{array}$ & 2 y (min) \\
\hline $\begin{array}{l}\text { Valdimarsson } \\
\text { et al. (1994) }\end{array}$ & Yes & (?) & No & $\begin{array}{l}\text { Serum } 25 \text {-hydroxy } \\
\text { vitamin } D\end{array}$ & 13 & & $\begin{array}{l}\text { Median: } 52 \\
\text { (range: } \\
\text { 18-91) }\end{array}$ & 17 & & $\begin{array}{l}\text { Median: } 80 \\
\text { (range: } 37-95 \text { ) }\end{array}$ & $\begin{array}{l}p<0.05 \\
\text { (favors } \\
\text { recovery) }\end{array}$ & $\begin{array}{l}8 \mathrm{y} \text { and } 9 \mathrm{y} \\
\text { (medians for } \\
\text { groups) with } \\
4-14 \mathrm{y} \text { (range) }\end{array}$ \\
\hline \multicolumn{13}{|c|}{ IRON AND FERRITIN } \\
\hline $\begin{array}{l}\text { Fang et al. } \\
(2017)\end{array}$ & No & $\mathrm{No}^{\mathrm{C}}$ & Yes & $\begin{array}{l}\text { Serum ferritin } \\
\text { (cut-off: males: } 24 \\
\text { mcg/l, females: } 11 \\
\text { mcg/l) }\end{array}$ & 69 & $7(10)$ & & 165 & $21(13)$ & & $p=0.6636$ & 2 y (min) \\
\hline $\begin{array}{l}\text { Kemppainen } \\
\text { et al. (1998) }\end{array}$ & No & Yes & (?) & $\begin{array}{l}\text { Serum total iron } \\
\text { (cut-off: not } \\
\text { reported) }\end{array}$ & 37 & $15(41)$ & & 3 & $0(0)$ & & Not reported & $1 \mathrm{y}$ \\
\hline $\begin{array}{l}\text { Kemppainen } \\
\text { et al. (1998) }\end{array}$ & No & Yes & (?) & $\begin{array}{l}\text { Serum ferritin } \\
\text { (cut-off: not } \\
\text { reported) }\end{array}$ & 37 & $5(14)$ & & 3 & $0(0)$ & & Not reported & $1 \mathrm{y}$ \\
\hline $\begin{array}{l}\text { Pekki et al. } \\
\text { (2015) }\end{array}$ & Yes & Yes & No & $\begin{array}{l}\text { Serum total iron } \\
(\mu \mathrm{mol} / \mathrm{l})\end{array}$ & $\sum 144^{a}$ & & $\begin{array}{l}\text { Median: } 19.6 \\
\left(Q_{1}-Q_{3}:\right. \\
13.4-24.4)\end{array}$ & $\sum 144^{\mathrm{a}}$ & & $\begin{array}{l}\text { Median: } 17.6 \\
\left(Q_{1}-Q_{3}:\right. \\
14.0-21.5)\end{array}$ & $p=0.245$ & $1 \mathrm{y}$ \\
\hline $\begin{array}{l}\text { Souroujon } \\
\text { et al. (1982) }\end{array}$ & Yes & Yes & (?) & Serum ferritin & 2 & & $\begin{array}{l}\text { Mean: } 16.2 \\
\text { (SD: } 1.38)\end{array}$ & 34 & & $\begin{array}{l}\text { Mean: 25.7 } \\
\text { (SD: 1.82) }\end{array}$ & Not reported & $1 \mathrm{y}$ \\
\hline \multicolumn{13}{|l|}{ CALCIUM } \\
\hline $\begin{array}{l}\text { Fang et al. } \\
(2017)\end{array}$ & No & $\mathrm{No}^{\mathrm{C}}$ & Yes & $\begin{array}{l}\text { Serum total } \\
\text { calcium (cut-off: } \\
8.9 \mathrm{mg} / \mathrm{dl} \text { ) }\end{array}$ & 65 & $13(20)$ & & 164 & $4(2)$ & & $\begin{array}{l}p<0.0001 \\
\text { (favors } \\
\text { recovery) }\end{array}$ & 2 y (min) \\
\hline
\end{tabular}


TABLE 7 | Continued

\begin{tabular}{|c|c|c|c|c|c|c|c|c|c|c|c|c|}
\hline \multirow[t]{2}{*}{ Study } & \multirow{2}{*}{$\begin{array}{l}\text { Parameter of } \\
\text { interest } \\
\text { assessed } \\
\text { prospectively? }\end{array}$} & \multirow{2}{*}{$\begin{array}{l}\text { Parameter of } \\
\text { interest } \\
\text { assessed at the } \\
\text { time of follow-up } \\
\text { biopsy? }\end{array}$} & \multirow{2}{*}{$\begin{array}{l}\text { Only } \\
\text { strict } \\
\text { GFD? }\end{array}$} & \multirow[t]{2}{*}{ Laboratory study } & \multicolumn{3}{|c|}{ Persistent villous atrophy } & \multicolumn{3}{|c|}{ Mucosal recovery } & \multirow{2}{*}{$\begin{array}{l}\text { Statistics } \\
\text { (atrophy vs. } \\
\text { recovery) }\end{array}$} & \multirow{2}{*}{$\begin{array}{l}\text { Timing of } \\
\text { follow-up } \\
\text { biopsy (from } \\
\text { CeD } \\
\text { diagnosis) }\end{array}$} \\
\hline & & & & & $\begin{array}{l}\text { Total } \mathbf{N}^{0} \text { of } \\
\text { pts }\end{array}$ & $\begin{array}{l}\mathbf{N}^{0} \text { of pts with } \\
\text { deficiency (\% of } \\
\text { total) }\end{array}$ & Level & $\begin{array}{l}\text { Total } \mathbf{N}^{0} \text { of } \\
\text { pts }\end{array}$ & $\begin{array}{l}\mathrm{N}^{0} \text { of pts with } \\
\text { deficiency (\% of } \\
\text { total) }\end{array}$ & Level & & \\
\hline $\begin{array}{l}\text { Pekki et al. } \\
\text { (2015) }\end{array}$ & Yes & Yes & No & $\begin{array}{l}\text { Serum ionized } \\
\text { calcium (mmol/l) }\end{array}$ & $\sum 95^{a}$ & & $\begin{array}{l}\text { Median: } 1.25 \\
\left(Q_{1}-Q_{3}:\right. \\
1.21-1.28)\end{array}$ & $\sum 95^{a}$ & & $\begin{array}{l}\text { Median: } 1.24 \\
\left(Q_{1}-Q_{3}:\right. \\
1.21-1.27)\end{array}$ & $p=0.708$ & $1 y$ \\
\hline $\begin{array}{l}\text { Valdimarsson } \\
\text { et al. (1994) }\end{array}$ & Yes & (?) & No & $\begin{array}{l}\text { Serum ionized } \\
\text { calcium (mmol/l) }\end{array}$ & 13 & & $\begin{array}{l}\text { Median: } 1.24 \\
\text { (range: } \\
1.16-1.31 \text { ) }\end{array}$ & 17 & & $\begin{array}{l}\text { Median: } 1.24 \\
\text { (range: } \\
1.19-1.34 \text { ) }\end{array}$ & $p=N S$ & $\begin{array}{l}8 \mathrm{y} \text { and } 9 \mathrm{y} \\
\text { (medians for } \\
\text { groups) with } \\
4-14 \text { y (range) }\end{array}$ \\
\hline \multicolumn{13}{|l|}{ ZINC } \\
\hline $\begin{array}{l}\text { Fang et al. } \\
\text { (2017) }\end{array}$ & No & $\mathrm{No}^{\mathrm{C}}$ & Yes & $\begin{array}{l}\text { serum zinc } \\
\text { (cut-off: } 0.66 \\
m c g / m l)\end{array}$ & 46 & $18(39)$ & & 145 & $20(14)$ & & $\begin{array}{l}p=0.0005 \\
\text { (favors } \\
\text { recovery) }\end{array}$ & 2 y (min) \\
\hline $\begin{array}{l}\text { Kemppainen } \\
\text { et al. (1998) }\end{array}$ & No & Yes & (?) & $\begin{array}{l}\text { serum zinc } \\
\text { (cut-off: not } \\
\text { reported) }\end{array}$ & 37 & $10(27)$ & & 3 & $0(0)$ & & Not reported & $1 \mathrm{y}$ \\
\hline \multicolumn{13}{|l|}{ COPPER } \\
\hline $\begin{array}{l}\text { Fang et al. } \\
\text { (2017) }\end{array}$ & No & $\mathrm{No}^{\mathrm{C}}$ & Yes & $\begin{array}{l}\text { Serum copper } \\
(0.75 \mathrm{mcg} / \mathrm{ml})\end{array}$ & 51 & $0(0)$ & & 149 & $4(3)$ & & $p=0.5740$ & 2 y $(\min )$ \\
\hline \multicolumn{13}{|c|}{ HOMOCYSTEINE } \\
\hline $\begin{array}{l}\text { Dickey et al. } \\
\text { (2008) }\end{array}$ & Yes & Yes & Yes & $\begin{array}{l}\text { Plasma } \\
\text { homocysteine } \\
(\mu \mathrm{mol} / \mathrm{l})\end{array}$ & 24 & & $\begin{array}{l}\text { Mean: } 11.4 \\
\text { (SD: } 5.5)\end{array}$ & 41 & & $\begin{array}{l}\text { Mean: } 10.4 \\
\text { (SD: 2.6) }\end{array}$ & Not reported & $1 \mathrm{y}(\mathrm{min})$ \\
\hline
\end{tabular}

${ }^{a}$ Only the total $N^{0}$ of pts was given. ${ }^{b}$ Parameter was measured years after the follow-up biopsy. ${ }^{c}$ Parameter was measured within 1 month of follow-up biopsy. ${ }^{d}$ Values were within the normal range for all patients and not reported for recovery and atrophic groups separately. (?) indicates uncertainty. CeD, celiac disease; EGRAC, erythrocyte glutathione reductase activation coefficient; GFD, gluten-free diet; $m$, month; min, minimum; NS, non-significant; $Q_{1}-Q_{3}, 25 \%$ and $75 \%$ quartiles; SD, standard deviation; $y$, year. 
TABLE 8 | Anemia and hemoglobin levels in celiac patients with persistent villous atrophy and mucosal recovery.

\begin{tabular}{|c|c|c|c|c|c|c|c|c|c|c|c|c|}
\hline \multirow[t]{2}{*}{ Study } & \multirow{2}{*}{$\begin{array}{l}\text { Parameter of } \\
\text { interest } \\
\text { assessed } \\
\text { prospectively? }\end{array}$} & \multirow{2}{*}{$\begin{array}{l}\text { Parameter of } \\
\text { interest } \\
\text { assessed at the } \\
\text { time of follow-up } \\
\text { biopsy? }\end{array}$} & \multirow{2}{*}{$\begin{array}{l}\text { Only } \\
\text { strict } \\
\text { GFD? }\end{array}$} & \multirow{2}{*}{$\begin{array}{l}\text { Laboratory } \\
\text { study }\end{array}$} & \multicolumn{3}{|c|}{ Persistent villous atrophy } & \multicolumn{3}{|c|}{ Mucosal recovery } & \multirow{2}{*}{$\begin{array}{l}\text { Statistics } \\
\text { (atrophy vs. } \\
\text { recovery) }\end{array}$} & \multirow{2}{*}{$\begin{array}{l}\text { Timing of } \\
\text { follow-up biopsy } \\
\text { (from CeD } \\
\text { diagnosis) }\end{array}$} \\
\hline & & & & & $\begin{array}{l}\text { Total } \mathbf{N}^{0} \text { of } \\
\text { pts }\end{array}$ & $\begin{array}{l}\mathrm{N}^{0} \text { of pts with } \\
\text { deficiency (\% of } \\
\text { total) }\end{array}$ & Level & $\begin{array}{l}\text { Total } \mathbf{N}^{0} \text { of } \\
\text { pts }\end{array}$ & $\begin{array}{l}\mathbf{N}^{0} \text { of pts with } \\
\text { deficiency }(\% \text { of } \\
\text { total) }\end{array}$ & Level & & \\
\hline $\begin{array}{l}\text { Cammarota } \\
\text { et al. (2007) }\end{array}$ & Yes & Yes & No & $\begin{array}{l}\text { Iron- } \\
\text { deficiency } \\
\text { anemia (Hb } \\
\text { cut-off: not } \\
\text { reported) }\end{array}$ & 49 & $16(33)$ & & 16 & $4(25)$ & & Not reported & 6-14 m (range) \\
\hline $\begin{array}{l}\text { Ciacci et al. } \\
(2002)\end{array}$ & No & Yes & No & $\mathrm{Hb}(\mathrm{g} / \mathrm{l})$ & 93 & & Mean: 133 & 297 & & Not reported ${ }^{a}$ & Not reported & 7.78 y (mean) \\
\hline $\begin{array}{l}\text { Fang et al. } \\
\text { (2017) }\end{array}$ & No & $\mathrm{No}$ & Yes & $\begin{array}{l}\text { anemia }(H b \\
\text { cut-off: } \\
\text { males: } 135 \\
\text { g/l, females: } \\
120 \mathrm{~g} / \mathrm{l})\end{array}$ & 97 & $14(14)$ & & 249 & $32(13)$ & & $p=0.7255$ & 2 y (min) \\
\hline $\begin{array}{l}\text { Kaukinen } \\
\text { et al. (2007) }\end{array}$ & Yes & Yes & Yes & $\mathrm{Hb}(\mathrm{g} / \mathrm{l})$ & 13 & & $\begin{array}{l}\text { mean: } 121 \\
\text { (Cl: 114-128) }\end{array}$ & 18 & & $\begin{array}{l}\text { Mean: } 13.2 \\
\text { (Cl: } \\
12.6-13.8)\end{array}$ & $p=\mathrm{NS}$ & $\begin{array}{l}8 \mathrm{y} \text { and } 10 \mathrm{y} \\
\text { (medians for } \\
\text { groups) with 3-30 } \\
\mathrm{y} \text { (range) }\end{array}$ \\
\hline $\begin{array}{l}\text { Kemppainen } \\
\text { et al. (1998) }\end{array}$ & No & Yes & (?) & $\begin{array}{l}\text { anemia (Hb } \\
\text { cut-off: not } \\
\text { reported) }\end{array}$ & 37 & 7 (19) & & 3 & 1 (33) & & Not reported & $1 \mathrm{y}$ \\
\hline $\begin{array}{l}\text { Mahadev } \\
\text { et al. (2017) }\end{array}$ & No & Yes & Yes & $\begin{array}{l}\text { anemia (Hb } \\
\text { cut-off: not } \\
\text { reported) }\end{array}$ & 511 & $114(22)$ & & 834 & $148(18)$ & & $\begin{array}{l}\text { unadjusted } \\
p=0.04 \\
\text { adjusted } \\
\boldsymbol{p}=\mathbf{0 . 2 3 6}^{\mathrm{c}}\end{array}$ & $\begin{array}{l}4 \text { y (median) with } 1 \\
y \text { (min) }\end{array}$ \\
\hline $\begin{array}{l}\text { Pekki et al. } \\
\text { (2015) }\end{array}$ & Yes & Yes & No & $\mathrm{Hb}(\mathrm{g} / \mathrm{l})$ & $\sum 153^{f}$ & & $\begin{array}{l}\text { Median: } 145 \\
\left(Q_{1}-Q_{3}:\right. \\
134-150)\end{array}$ & $\sum 153^{f}$ & & $\begin{array}{l}\text { Median: } 147 \\
\left(Q_{1}-Q_{3}:\right. \\
147-151)\end{array}$ & $p=0.342$ & $1 y$ \\
\hline $\begin{array}{l}\text { Thornquist } \\
\text { et al. (1993) }\end{array}$ & Yes & $\mathrm{No}^{d}$ & No & $\mathrm{Hb}$ & 4 & & Not reportede & 9 & & Not reportede & Not reported & $\begin{array}{l}17 \text { y (mean) with } \\
15-18 \text { y (range ?) }\end{array}$ \\
\hline $\begin{array}{l}\text { Tuire et al. } \\
\text { (2012) }\end{array}$ & Yes & Yes & Yes & $\mathrm{Hb}(\mathrm{g} / \mathrm{l})$ & 7 & & $\begin{array}{l}\text { Median: } 140 \\
\text { (range: } \\
128-161 \text { ) }\end{array}$ & 170 & & Not reported ${ }^{a}$ & Not reported & $2-41$ y (range) \\
\hline
\end{tabular}

Multivariate analyses are highlighted with bold. ${ }^{a}$ Values were given by Marsh 0 and 1-2 separately. ${ }^{b}$ Parameter was assessed within 1-month of follow-up biopsy. ${ }^{~}$ The analysis was adjusted for age, gender, body mass index, duration of GFD, medications, laboratory tests, and symptoms. ${ }^{d}$ Parameter was measured years after the follow-up biopsy. ${ }^{e}$ Values by recovery and atrophy were not reported separately. (?) indicates uncertainty. CeD, celiac disease; Cl:

confidence interval; GFD, gluten-free diet; Hb, hemoglobin; $m$, month; min, minimum; NS, non-significant; $Q_{1}-Q_{3}, 25 \%$ and $75 \%$ quartiles; SD, standard deviation; $y$, year. 

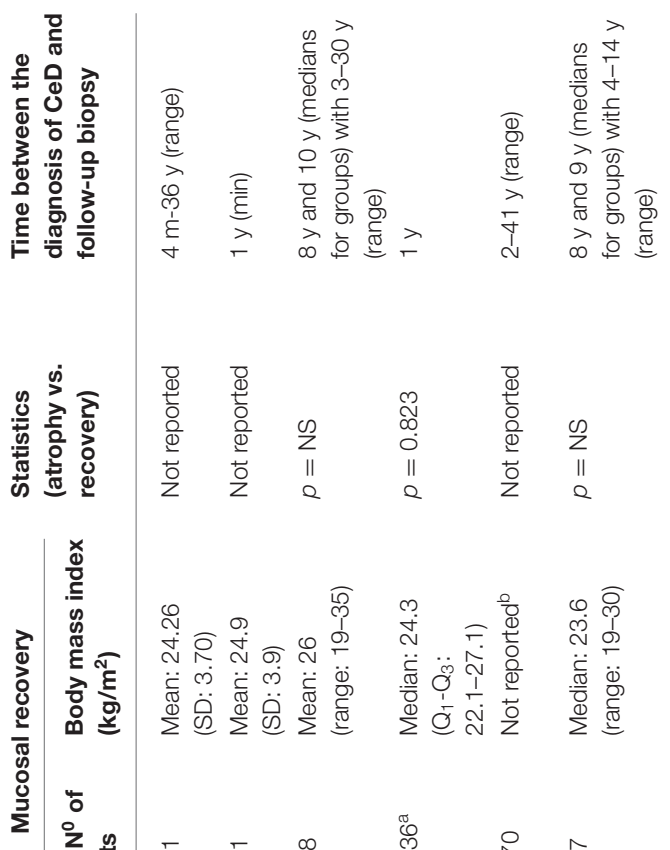

pneumococcal, influenza, herpes zoster, and Clostridium difficile infections) (Emilsson et al., 2018) and adverse pregnancy outcomes (Lebwohl et al., 2015b) were not influenced by follow-up histology. However, there was a significant reduction in the frequency of respiratory and dermatological diseases (Pekki et al., 2017) and in the rate of elevated aspartate and alanine transaminase levels with MR (Mahadev et al., 2017). In contrast, MR predisposed to developing anxiety and depression (Ludvigsson et al., 2018) as well as epilepsy (Kurien et al., 2018). The effect of follow-up histology on immune-mediated co-morbidities including dermatitis herpetiformis is severely understudied (Valdimarsson et al., 1994; Tuire et al., 2012; Mahadev et al., 2017).

\section{All-Cause and Cause-Specific Mortality}

Four studies reported all-cause mortality during follow-up (Kaukinen et al., 2007; Rubio-Tapia et al., 2010; Lebwohl et al., 2013a; Pekki et al., 2015), two of which adjusted the analysis for significant co-variates (Rubio-Tapia et al., 2010; Lebwohl et al., 2013a). In this regard, MR seemed to be protective in a small retrospective study with a HR of 0.13 (CI: 0.02-1.06) (RubioTapia et al., 2010), not confirmed by a nationwide registry analysis (HR $=1.0$, CI: $0.86-1.19$ for PVA) (Lebwohl et al., 2013a). In the latter study, results were consistent on cardiovascular, tumorrelated and respiratory deaths: the beneficial effect of MR was not confirmed (Lebwohl et al., 2013a) (Table 11).

\section{DISCUSSION}

\section{Summary of Findings}

Whether the follow-up biopsy in asymptomatic $\mathrm{CeD}$ is needed is uncertain, and, to date, no systematic review has addressed this question. Achieving MR is a desirable goal of treatment; however, findings of reports which investigated its beneficial effects on disease course are inconsistent. In line with our previous findings, MR rates ranged from 9 to $97 \%$ across the studies included (Szakacs et al., 2017). The fact that a considerable fraction of $\mathrm{CeD}$ patients does not achieve MR underlines the importance of investigating the potential prognostic role of follow-up histology.

One important conclusion of this review is that many asymptomatic patients do not achieve MR, and vice versa, many symptomatic patients do achieve MR. Analogously, MR cannot guarantee the symptoms to disappear, whereas PVA is often associated with persisting symptoms, even on a long-term followup exceeding 1 year (Tables 2, 3). Persisting symptoms may indicate poor dietary adherence (Abdulkarim et al., 2002; Leffler et al., 2007; Haere et al., 2016) or gluten-independent food intolerance (Carroccio et al., 2008) but other diseases, such as pancreatic insufficiency or small intestinal bacterial overgrowth, might be in the background (Fine et al., 1997). Noteworthy that a few studies included patients with questionable adherence to gluten-free diet, although a top-quality study established a straight relationship between persisting symptoms and PVA in co-variate-adjusted analysis of patients with good adherence (Carroccio et al., 2008). Studies used various definitions for assessing symptoms; some did not report how symptoms were specified at all. Although a smaller study favored MR 
TABLE 10 | Malignant tumors in celiac patients with persistent villous atrophy and mucosal recovery.

\begin{tabular}{|c|c|c|c|c|c|c|c|}
\hline \multirow[t]{2}{*}{ Study } & \multirow{2}{*}{$\begin{array}{l}\text { Only } \\
\text { strict } \\
\text { GFD? }\end{array}$} & \multirow[t]{2}{*}{$\begin{array}{l}\text { Malignant } \\
\text { tumors }\end{array}$} & \multicolumn{2}{|c|}{$\begin{array}{c}\mathrm{N}^{0} \text { of cases/Total } \\
(\%)\end{array}$} & \multirow[t]{2}{*}{ Statistics } & \multirow{2}{*}{$\begin{array}{l}\text { Timing of follow-up } \\
\text { biopsy } \\
\text { (from diagnosis of } \\
\text { CeD) }\end{array}$} & \multirow[t]{2}{*}{ Follow-up period } \\
\hline & & & $\begin{array}{l}\text { Persistent } \\
\text { villous atrophy }\end{array}$ & $\begin{array}{l}\text { Mucosal } \\
\text { recovery }\end{array}$ & & & \\
\hline $\begin{array}{l}\text { Kaukinen et al. } \\
\text { (2007) }\end{array}$ & Yes & $\begin{array}{l}\text { Any malignant } \\
\text { tumor }^{\mathrm{a}}\end{array}$ & $\begin{array}{l}3 \text { cases/13 pts } \\
(23.1)\end{array}$ & $\begin{array}{l}0 \text { cases } / 18 \text { pts } \\
(0.0)\end{array}$ & Not reported & $\begin{array}{l}8 \text { y and } 10 \text { y (medians } \\
\text { for groups) (range: } \\
3-30 \text { y) }\end{array}$ & $\begin{array}{l}\text { 4-5 years from } \\
\text { follow-up biopsy }\end{array}$ \\
\hline \multirow[t]{4}{*}{$\begin{array}{l}\text { Lebwohl et al. } \\
\text { (2013b) }\end{array}$} & No & Any lymphoma & $\begin{array}{l}41 \text { cases/4317 pts } \\
(0.9)\end{array}$ & $\begin{array}{l}12 \text { cases } / 3308 \text { pts } \\
(0.4)\end{array}$ & $\begin{array}{l}\mathrm{HR}=2.26 \\
(\mathrm{Cl}: 2.28-4.34)^{\mathrm{d}}\end{array}$ & $\begin{array}{l}1.3 \text { (median) (range: } 6 \\
\mathrm{~m}-5 \mathrm{y})\end{array}$ & \multirow{4}{*}{$\begin{array}{l}10.6 \text { y (median) } \\
\text { from diagnosis of } \\
\text { CeD and } 8.9 \text { y } \\
\text { (median) from } \\
\text { follow-up biopsy }\end{array}$} \\
\hline & & $\begin{array}{l}\text { Any non-Hodgkin } \\
\text { lymphoma }\end{array}$ & $\begin{array}{l}34 \text { cases/4317 pts } \\
(0.8)\end{array}$ & $\begin{array}{l}8 \text { cases } / 3308 \text { pts } \\
(0.2)\end{array}$ & $\begin{array}{l}\mathrm{HR}=2.82 \\
(\mathrm{Cl}: 1.29-6.17)^{\mathrm{d}}\end{array}$ & & \\
\hline & & $\begin{array}{l}\text { Any B-cell } \\
\text { lymphoma }\end{array}$ & $\begin{array}{l}4 \text { cases } / 4317 \text { pts } \\
(<0.1)\end{array}$ & $\begin{array}{l}3 \text { cases } / 3308 \text { pts } \\
(<0.1)\end{array}$ & $\begin{array}{l}\text { HR }=0.97 \\
(\mathrm{Cl}: 0.21-4.49)^{d}\end{array}$ & & \\
\hline & & $\begin{array}{l}\text { Any T-cell } \\
\text { lymphoma }\end{array}$ & $\begin{array}{l}10 \text { cases } / 4317 \text { pts } \\
(0.2)\end{array}$ & $\begin{array}{l}2 \text { cases } / 3308 \text { pts } \\
(<0.1)\end{array}$ & $\begin{array}{l}H R=3.51 \\
(C I: 0.75-16.34)^{d}\end{array}$ & & \\
\hline Pekki et al. (2015) & No & $\begin{array}{l}\text { Any malignant } \\
\text { tumor }\end{array}$ & $\begin{array}{l}9 \text { cases/200 pts } \\
(4.5)\end{array}$ & $\begin{array}{l}14 \text { cases/276 pts } \\
(5.1)\end{array}$ & $p=0.762$ & $1 \mathrm{y}$ & $\begin{array}{l}8 \text { y (median) from } \\
\text { diagnosis of CeD }\end{array}$ \\
\hline \multirow[t]{2}{*}{ Pekki et al. (2017) } & No & $\begin{array}{l}\text { Any malignant } \\
\text { tumor }{ }^{b}\end{array}$ & $\begin{array}{l}8 \text { cases/71 pts } \\
(11.3)\end{array}$ & $\begin{array}{l}8 \text { cases/134 pts } \\
(6.0)\end{array}$ & $p=0.116$ & $1 y$ & $\begin{array}{l}5 \text { y (median) from } \\
\text { diagnosis of } \mathrm{CeD}\end{array}$ \\
\hline & & Any lymphoma & $\begin{array}{l}1 \text { case/71 pts } \\
(1.4)\end{array}$ & $\begin{array}{l}2 \text { cases/134 pts } \\
(1.5)\end{array}$ & $p=0.968$ & & \\
\hline Tuire et al. (2012) & Yes & $\begin{array}{l}\text { Any malignant } \\
\text { tumor }^{c}\end{array}$ & $\begin{array}{l}0 \text { cases } / 7 \mathrm{pts} \\
(0.0)\end{array}$ & $\begin{array}{l}4 \text { cases } / 170 \text { pts } \\
(2.4)\end{array}$ & Not reported & $\begin{array}{l}7 \mathrm{y}, 9 \mathrm{y}, \text { and } 10 \mathrm{y} \\
\text { (medians for groups) } \\
\text { (range: } 2-41 \mathrm{y} \text { ) }\end{array}$ & $\begin{array}{l}\text { From diagnosis of } \\
\text { CeD until } \\
\text { follow-up biopsy }\end{array}$ \\
\hline
\end{tabular}

Multivariate analyses are highlighted with bold. ${ }^{a}$ Cases included T-cell lymphoma, small bowel adenocarcinoma, and carcinoid tumor; ${ }^{b}$ Cases included the uterus, breast, lung, pancreas,

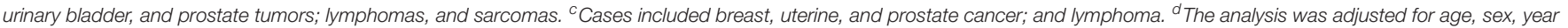

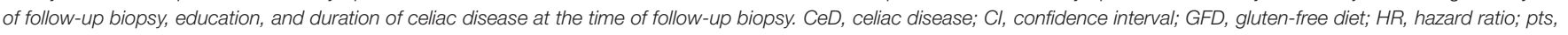
patients; $m$, month; $y$, year.

regarding a set of individual symptoms (Carroccio et al., 2008), the most extensive study including only symptomatic patients found significant associations in univariate analysis (with an unexpected inverse association between PVA and heartburn). Interestingly, all associations proved to be nonsignificant after adjusting for co-variates (Mahadev et al., 2017). Self-reporting of subjective complaints may contribute to the discrepant results. Use of symptom scales (Svedlund et al., 1988) provided a comparable measure with homogenous results: none of the studies attributed higher scores for those patients with PVA compared to those with MR (Table 4). The same association applies to quality of life; however, based on small sets of patients (Table 4). Altogether, the diagnostic accuracy of persisting symptoms seems insufficient for indicating a follow-up biopsy. Evidence coming from the two prospective studies is not enough to decide whether PVA precisely predicts the long-term persistence of symptoms (Pekki et al., 2015, 2017) (Table 4).

PVA appears to be associated with radiologically detected metabolic bone disease in univariate analysis (Table 5), which translates into an increased risk of hip fractures (as an independent predictor) but not into that of the overall fractures (Table 6). Calcium malabsorption (Fang et al., 2017) together with vitamin D deficiency (Valdimarsson et al., 1994; Fang et al., 2017) are likely to be causative factors. Length of follow-up (not standardized among studies) may be insufficient for restoring bone mineral density (Pekki et al., 2015), especially in those with severe bone impairment at diagnosis. Body mass index, as an important co-variate, seems to be independent of mucosal status (Table 9).

Laboratory studies describing vitamin and mineral levels tend to recover on an adequate diet. Patients on a long-term strict gluten-free diet may suffer from vitamin deficiencies (Hallert et al., 2002, 2009), this phenomenon might be explained by the vitamin- and micronutrient-poor nature of the gluten-free diet as compared to a balanced gluten-containing diet (Thompson, 1999). None of the studies adjusted for dietary adherence and vitamin supplementation which might counteract malabsorption and masked the effects of PVA. Variability in clinical and histological severity at diagnosis might delay villous restitution, thereby affecting vitamin status (not taken into account in the studies) (Kemppainen et al., 1998). Besides, improvement of histology might lag behind the recovery of laboratory values (Pekki et al., 2015), although the length of follow-up exceeded 1 year in all studies (Table 7).

Regarding mortality, the adjusted HRs calculated in the individual studies attributed a neutral effect to MR and PVA (Table 11) (Rubio-Tapia et al., 2010; Lebwohl et al., 2013a). Findings were similar on malignant tumors, except in certain lymphoproliferative diseases based on an extensive registry analysis (noteworthy that data were not adjusted for dietary adherence) (Lebwohl et al., 2013b). A possible explanation could be that the increment in rates of mortality and tumors with PVA 
TABLE 11 | Studies reporting on mortality in celiac patients with persistent villous atrophy and mucosal recovery.

\begin{tabular}{|c|c|c|c|c|c|c|c|}
\hline \multirow[t]{2}{*}{ Study } & \multirow{2}{*}{$\begin{array}{l}\text { Only } \\
\text { strict } \\
\text { GFD? }\end{array}$} & \multirow[t]{2}{*}{ Mortality } & \multicolumn{2}{|c|}{$\begin{array}{c}\mathrm{N}^{0} \text { of cases/Total } \\
(\%)\end{array}$} & \multirow{2}{*}{$\begin{array}{l}\text { Statistics } \\
\text { (atrophy vs. } \\
\text { recovery) }\end{array}$} & \multirow{2}{*}{$\begin{array}{l}\text { Timing of follow-up } \\
\text { biopsy } \\
\text { (from diagnosis of } \\
\text { CeD) }\end{array}$} & \multirow[t]{2}{*}{ Follow-up period } \\
\hline & & & $\begin{array}{l}\text { Persistent } \\
\text { villous atrophy }\end{array}$ & $\begin{array}{l}\text { Mucosal } \\
\text { recovery }\end{array}$ & & & \\
\hline $\begin{array}{l}\text { Kaukinen et al. } \\
\text { (2007) }\end{array}$ & Yes & All-cause & $\begin{array}{l}2 \text { cases } / 13 \text { pts } \\
(15.4)\end{array}$ & $\begin{array}{l}1 \text { case/18 pts } \\
(5.6)\end{array}$ & Not reported & $\begin{array}{l}8 \text { y and } 10 \text { y (medians } \\
\text { for groups) (range: } \\
3-30 \text { y) }\end{array}$ & $\begin{array}{l}4-5 \text { years from follow-up } \\
\text { biopsy }\end{array}$ \\
\hline Pekki et al. (2015) & No & All-cause & $\begin{array}{l}10 \text { cases/71 pts } \\
(14.1)\end{array}$ & $\begin{array}{l}12 \text { cases } / 134 \text { pts } \\
(9.0)\end{array}$ & $p=0.259$ & $1 \mathrm{y}$ & $\begin{array}{l}8 \text { y (median) from diagnosis } \\
\text { of } \mathrm{CeD}\end{array}$ \\
\hline $\begin{array}{l}\text { Rubio-Tapia et al. } \\
\text { (2010) }\end{array}$ & No & All-cause & $\begin{array}{l}10 \text { cases } / 117 \text { pts } \\
(8.5)\end{array}$ & $\begin{array}{l}1 \text { case } / 124 \text { pts } \\
(0.8)\end{array}$ & $\begin{array}{l}\mathrm{HR}=0.13 \\
(\mathrm{Cl}: 0.02-1.06)^{\mathrm{a}} \\
p=0.06 \\
\text { (favors } \\
\text { recovery) }\end{array}$ & Min: 5 m & 10 y from diagnosis of $\mathrm{CeD}$ \\
\hline \multirow[t]{4}{*}{$\begin{array}{l}\text { Lebwohl et al. } \\
\text { (2013a) }\end{array}$} & No & $\begin{array}{l}\text { All-cause } \\
\text { Cardiovascular } \\
\text { Malignancy } \\
\text { Respiratory }\end{array}$ & $\begin{array}{l}\text { Unknown number } \\
\text { of } 3,317 \text { pts }^{b}\end{array}$ & $\begin{array}{l}\text { Unknown number } \\
\text { of } 4,331 \text { pts }^{\text {b }}\end{array}$ & 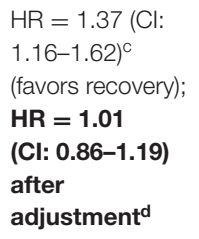 & $\begin{array}{l}1.3 \text { (median) (range: } 6 \\
\mathrm{~m}-5 \mathrm{y})\end{array}$ & $\begin{array}{l}11.5 \text { y (median) from } \\
\text { diagnosis of CeD and } 9.9 \text { y } \\
\text { (median) from follow-up } \\
\text { biopsy }\end{array}$ \\
\hline & & & & & $\begin{array}{l}\mathrm{HR}=1.03 \\
(\mathrm{Cl}: 0.76-1.38)^{\mathrm{d}}\end{array}$ & & \\
\hline & & & & & $\begin{array}{l}\mathrm{HR}=1.20 \\
(\mathrm{Cl}: 0.88-1.66)^{\mathrm{d}}\end{array}$ & & \\
\hline & & & & & $\begin{array}{l}\mathrm{HR}=0.78 \\
(\mathrm{Cl}: 0.41-1.48)^{\mathrm{d}}\end{array}$ & & \\
\hline
\end{tabular}

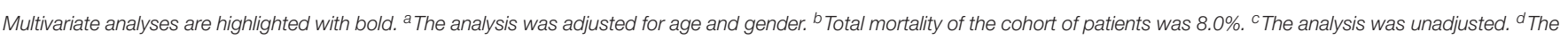

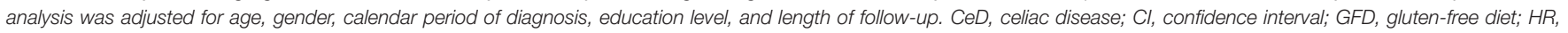
hazard ratio; pts, patients; $m$, month; $y$, year.

was counteracted by a decreased risk of ovarian, endometrial and breast cancer, likely due to lower body weight and/or earlier menopause (Ludvigsson et al., 2012; Lebwohl et al., 2013a).

\section{Strengths and Limitations}

Our study has several strengths. The question we raised is unique without known previous systematic review. We carried out an extensive systematic search with high coverage of patientimportant outcomes in a transparent manner by independent (and pre-trained) investigators with good inter-rater agreement. The quality of the included papers was rigorously assessed.

Although the amount of data presented in the tables would be enough for meta-analytical aggregations that we intended to do initially (as declared in the protocol of the work, see the PROSPERO record); finally, our team decided not to do so because of several concerns. The decision-making algorithm is presented in Figure 2.

We are aware that the evidence suffers from several limitations. (1) Most studies did not recruit patients immediately after the diagnosis of $\mathrm{CeD}(29 / 31,94 \%)$, did not take the followup biopsy after recruitment $(23 / 31,74 \%)$ and did not assess the disease course prospectively. (2) Although the diagnosis of $\mathrm{CeD}$ was mainly biopsy-proven, only two papers included newly diagnosed cases and observed them longitudinally until the follow-up biopsy. (3) Details of histological sampling and processing (e.g., sampling site, number of tissue samples, orientation, staining) were not consistently reported across papers, while the procedures often deviated from recent gold standards (e.g., samples were taken from the jejunum, histological classification systems were not specified). Similarly, the definition of villous atrophy varied across the studies. (4) Observational studies are vulnerable to bias: in registry analyses, data of the deceased were often missing (survivorship bias), attendance to regular control visits was incomplete (attrition bias), baseline differences between groups of patients with MR and those with PVA were rarely balanced with co-variateadjusted analyses (selection bias), study samples were not taken consistently from the general $\mathrm{CeD}$ population with consecutive recruitment (selection bias), and investigators assessing the outcomes were rarely blind to mucosal status (performance bias, detection bias). (5) Most evidence came from studies focusing on adults while an inverse association between age and rate of $\mathrm{MR}$ is well-known (Szakacs et al., 2017). (6) A strict gluten-free diet was not a criterion in several studies, the length of diet varied. (7) Publication bias cannot be reliably assessed in systematic reviews.

\section{CONCLUSION}

\section{Implications for Practice}

The results of publications on the prognostic role of follow-up histology (that is, MR and PVA) are not in agreement. Some adverse outcomes (e.g., persistent symptoms and metabolic bone 


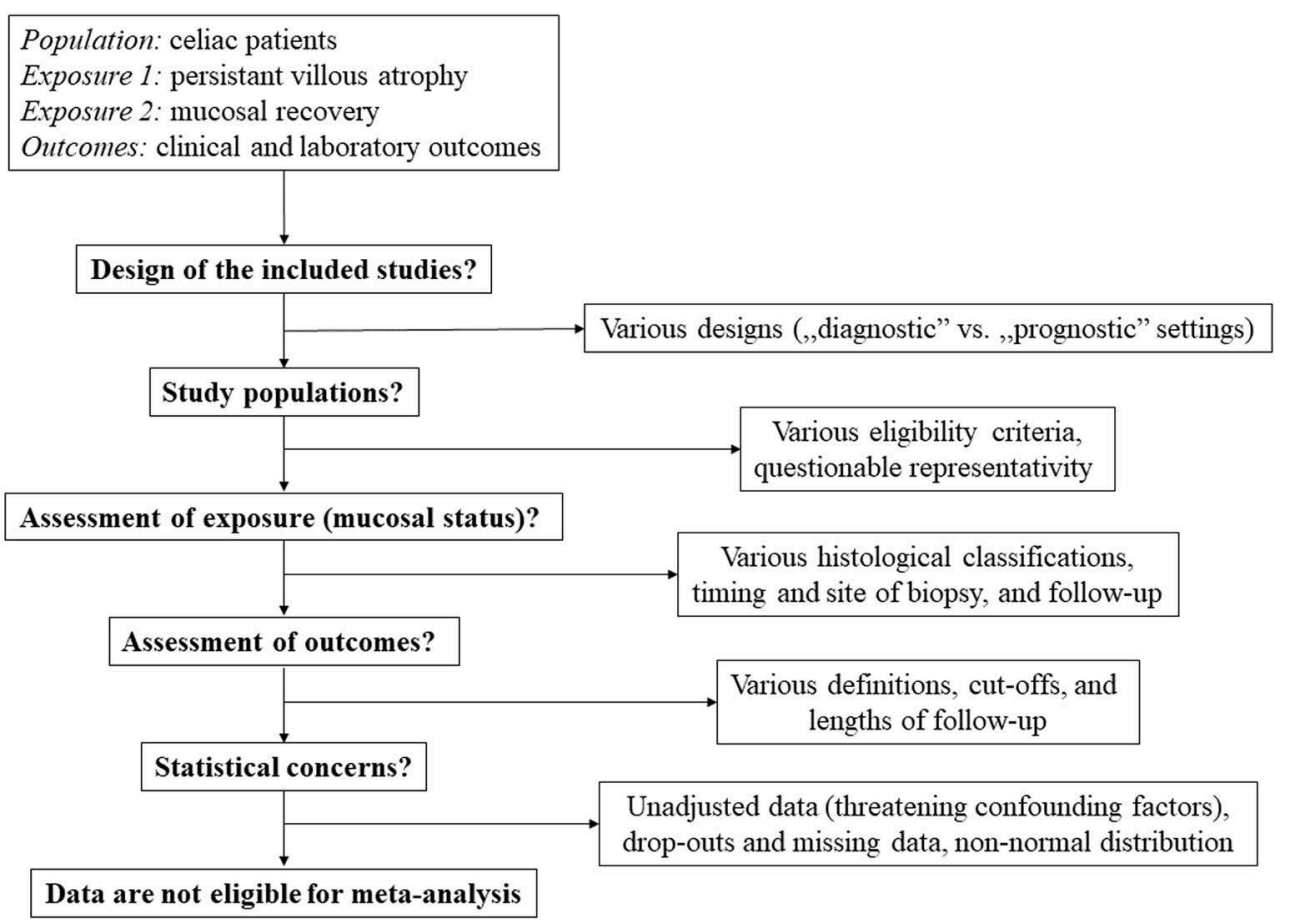

FIGURE 2 | Decision-making algorithm. Based on several arguments, we decided not to perform a meta-analysis.

disease) may be more common with PVA; however, achieving MR alone cannot guarantee a favorable clinical course to our current knowledge. The question as to whether taking a follow-up biopsy is beneficial has remained a matter of debate.

\section{Implications for Research}

With a view to the future, prospective cohort studies are urged to be organized to collect decisive evidence on the prognostic role of follow-up histology.

\section{DATA AVAILABILITY STATEMENT}

All datasets analyzed this study are included in the manuscript/supplementary files.

\section{AUTHOR CONTRIBUTIONS}

ZS and JB conceptualized the study. AM, DD, VB, LS, $\mathrm{AV}$, and $\mathrm{RH}$ collected the data. NG performed the formal

\section{REFERENCES}

Abdulkarim, A. S., Burgart, L. J., See, J., and Murray, J. A. (2002). Etiology of nonresponsive celiac disease: results of a systematic approach. Am. J. Gastroenterol. 97, 2016-2021. doi: 10.1111/j.1572-0241.2002.05917.x

Bai, J. C., Fried, M., Corazza, G. R., Schuppan, D., Farthing, M., Catassi, C., et al. (2013). World gastroenterology organisation global guidelines on celiac disease. J. Clin. Gastroenterol. 47, 121-126. doi: 10.1097/MCG.0b013e31827a6f83 analysis and designed the figures. ZS assessed the quality of studies. ZG, DC, and MS interpreted the results. ÁV, ZS, $\mathrm{JB}$, and BE drafted the manuscript. ÁV and $\mathrm{PH}$ supervised the work. All authors approved the final draft submitted, involved in the study design, edited, read and approved the final manuscript.

\section{FUNDING}

This study was supported by an Economic Development and Innovation Operative Programme Grant (GINOP 2.3.2-152016-00048), an Institutional Development for Enhancing Intelligent Specialization Grant (EFOP-3.6.2-16-2017-0006) of the National Research, Development and Innovation Office, the ÚNKP-18-3-I and ÚNKP-19-3-I New National Excellence Program of the Ministry of Human Capacities. Sponsors had no effect on study design, data collection, analysis, interpretation of the findings, or preparation of the manuscript. 
Cammarota, G., Cuoco, L., Cesaro, P., Santoro, L., Cazzato, A., Montalto, M., et al. (2007). A highly accurate method for monitoring histological recovery in patients with celiac disease on a gluten-free diet using an endoscopic approach that avoids the need for biopsy: a double-center study. Endoscopy 39, 46-51. doi: 10.1055/s-2006-945044

Carroccio, A., Ambrosiano, G., Di Prima, L., Pirrone, G., Iacono, G., Florena, A. M., et al. (2008). Clinical symptoms in celiac patients on a gluten-free diet. Scand. J. Gastroenterol. 43, 1315-1321. doi: 10.1080/00365520802200044

Catassi, C., Gatti, S., and Fasano, A. (2014). The new epidemiology of celiac disease. J. Pediatr. Gastroenterol. Nutr. 59 (Suppl. 1), S7-9. doi: 10.1097/01.mpg.0000450393.23156.59

Ciacci, C., Cirillo, M., Cavallaro, R., and Mazzacca, G. (2002). Long-term followup of celiac adults on gluten-free diet: prevalence and correlates of intestinal damage. Digestion 66, 178-185. doi: 10.1159/000066757

Corazza, G. R., and Villanacci, V. (2005). Coeliac disease. J. Clin. Pathol. 58, 573-574. doi: 10.1136/jcp.2004.023978

Cornell, H. J., Czyzewska, A., Macrae, F. A., Rydzewska, G., NasierowskaGutmejer, A., Bednarczuk, A., et al. (2016). The effect of enzyme supplementation on symptoms and duodenal histology in celiac patients. Int. J. Cell. Dis. 4, 40-47. doi: 10.12691/ijcd-4-2-2

Di Sabatino, A., and Corazza, G. R. (2009). Coeliac disease. Lancet 373, 1480-1493. doi: 10.1016/S0140-6736(09)60254-3

Dickey, W., Ward, M., Whittle, C. R., Kelly, M. T., Pentieva, K., Horigan, G., et al. (2008). Homocysteine and related B-vitamin status in coeliac disease: effects of gluten exclusion and histological recovery. Scand. J. Gastroenterol. 43, 682-688. doi: 10.1080/00365520701881118

Emilsson, L., Lebwohl, B., Green, P. H., Murray, J. A., Marild, K., and Ludvigsson, J. F. (2018). Mucosal healing and the risk of serious infections in patients with celiac disease. United European Gastroenterol. J. 6, 55-62. doi: $10.1177 / 2050640617707868$

Fang, H., King, K. S., Larson, J. J., Snyder, M. R., Wu, T. T., Gandhi, M. J., et al. (2017). Undetectable negative tissue transglutaminase IgA antibodies predict mucosal healing in treated coeliac disease patients. Aliment. Pharmacol. Ther. 46, 681-687. doi: 10.1111/apt.14250

Fine, K. D., Meyer, R. L., and Lee, E. L. (1997). The prevalence and causes of chronic diarrhea in patients with celiac sprue treated with a glutenfree diet. Gastroenterology 112, 1830-1838. doi: 10.1053/gast.1997.v112.pm91 78673

Ghazzawi, Y., Rubio-Tapia, A., Murray, J. A., and Absah, I. (2014). Mucosal healing in children with treated celiac disease. J. Pediatr. Gastroenterol. Nutr. 59, 229-231. doi: 10.1097/MPG.0000000000000390

Haere, P., Hoie, O., Schulz, T., Schonhardt, I., Raki, M., and Lundin, K. E. (2016). Long-term mucosal recovery and healing in celiac disease is the rule - not the exception. Scand. J. Gastroenterol. 51, 1439-1446. doi: 10.1080/00365521.2016.1218540

Haines, M. L., Anderson, R. P., and Gibson, P. R. (2008). Systematic review: the evidence base for long-term management of coeliac disease. Aliment. Pharmacol. Ther. 28, 1042-1066. doi: 10.1111/j.1365-2036.2008.03820.x

Hallert, C., Grant, C., Grehn, S., Granno, C., Hulten, S., Midhagen, G., et al. (2002). Evidence of poor vitamin status in coeliac patients on a gluten-free diet for 10 years. Aliment. Pharmacol. Ther. 16, 1333-1339. doi: 10.1046/j.1365-2036.2002.01283.x

Hallert, C., Svensson, M., Tholstrup, J., and Hultberg, B. (2009). Clinical trial: B vitamins improve health in patients with coeliac disease living on a gluten-free diet. Aliment. Pharmacol. Ther. 29, 811-816. doi: 10.1111/j.1365-2036.2009.03945.x

Hayden, J. A., Cote, P., and Bombardier, C. (2006). Evaluation of the quality of prognosis studies in systematic reviews. Ann. Intern. Med. 144, 427-437. doi: 10.7326/0003-4819-144-6-200603210-00010

Kaukinen, K., Peraaho, M., Lindfors, K., Partanen, J., Woolley, N., Pikkarainen, P., et al. (2007). Persistent small bowel mucosal villous atrophy without symptoms in coeliac disease. Aliment. Pharmacol. Ther. 25, 1237-1245. doi: 10.1111/j.1365-2036.2007.03311.x

Kemppainen, T. A., Kosma, V. M., Janatuinen, E. K., Julkunen, R. J., Pikkarainen, P. H., and Uusitupa, M. I. (1998). Nutritional status of newly diagnosed celiac disease patients before and after the institution of a celiac disease dietassociation with the grade of mucosal villous atrophy. Am. J. Clin. Nutr. 67, 482-487. doi: 10.1093/ajcn/67.3.482
Koskinen, O., Collin, P., Lindfors, K., Laurila, K., Maki, M., and Kaukinen, K. (2010). Usefulness of small-bowel mucosal transglutaminase-2 specific autoantibody deposits in the diagnosis and follow-up of celiac disease. J. Clin. Gastroenterol. 44, 483-488. doi: 10.1097/MCG.0b013e3181b64557

Kurien, M., Ludvigsson, J. F., Sanders, D. S., Zylberberg, H. M., Green, P. H., Sundelin, H. E. K., et al. (2018). Persistent mucosal damage and risk of epilepsy in people with celiac disease. Eur. J. Neurol. 25, 592-e538. doi: 10.1111/ene.13564

Lebwohl, B., Emilsson, L., Frobert, O., Einstein, A. J., Green, P. H., and Ludvigsson, J. F. (2015a). Mucosal healing and the risk of ischemic heart disease or atrial fibrillation in patients with celiac disease; a population-based study. PLoS ONE 10:e0117529. doi: 10.1371/journal.pone.0117529

Lebwohl, B., Granath, F., Ekbom, A., Montgomery, S. M., Murray, J. A., RubioTapia, A., et al. (2013a). Mucosal healing and mortality in coeliac disease. Aliment. Pharmacol. Ther. 37, 332-339. doi: 10.1111/apt.12164

Lebwohl, B., Granath, F., Ekbom, A., Smedby, K. E., Murray, J. A., Neugut, A. I., et al. (2013b). Mucosal healing and risk for lymphoproliferative malignancy in celiac disease: a population-based cohort study. Ann. Intern. Med. 159, 169-175. doi: 10.7326/0003-4819-159-3-201308060-00006

Lebwohl, B., Michaelsson, K., Green, P. H., and Ludvigsson, J. F. (2014). Persistent mucosal damage and risk of fracture in celiac disease. J. Clin. Endocrinol. Metab. 99, 609-616. doi: 10.1210/jc.2013-3164

Lebwohl, B., Stephansson, O., Green, P. H., and Ludvigsson, J. F. (2015b). Mucosal healing in patients with celiac disease and outcomes of pregnancy: a nationwide population-based study. Clin. Gastroenterol. Hepatol. 13, 1111-1117.e1112. doi: 10.1016/j.cgh.2014.11.018

Leffler, D. A., Dennis, M., Hyett, B., Kelly, E., Schuppan, D., and Kelly, C. P. (2007). Etiologies and predictors of diagnosis in non-responsive celiac disease. Clin. Gastroenterol. Hepatol. 5, 445-450. doi: 10.1016/j.cgh.2006. 12.006

Leonard, M. M., Weir, D. C., Degroote, M., Mitchell, P. D., Singh, P., Silvester, J. A., et al. (2017). Value of IgA tTG in predicting mucosal recovery in children with celiac disease on a gluten-free diet. J. Pediatr. Gastroenterol. Nutr. 64, 286-291. doi: 10.1097/MPG.0000000000001460

Ludvigsson, J. F., Bai, J. C., Biagi, F., Card, T. R., Ciacci, C., Ciclitira, P. J., et al. (2014). Diagnosis and management of adult coeliac disease: guidelines from the British Society of Gastroenterology. Gut 63, 1210-1228. doi: 10.1136/gutjnl-2013-306578

Ludvigsson, J. F., Lebwohl, B., Chen, Q., Broms, G., Wolf, R. L., Green, P. H. R., et al. (2018). Anxiety after coeliac disease diagnosis predicts mucosal healing: a population-based study. Aliment. Pharmacol. Ther. 48, 1091-1098. doi: 10.1111/apt.14991

Ludvigsson, J. F., West, J., Ekbom, A., and Stephansson, O. (2012). Reduced risk of breast, endometrial and ovarian cancer in women with celiac disease. Int. J. Cancer 131, E244-250. doi: 10.1002/ijc.26454

Mahadev, S., Murray, J. A., Wu, T. T., Chandan, V. S., Torbenson, M. S., Kelly, C. P., et al. (2017). Factors associated with villus atrophy in symptomatic coeliac disease patients on a gluten-free diet. Aliment. Pharmacol. Ther. 45, 1084-1093. doi: 10.1111/apt.13988

Marsh, M. N. (1992). Gluten, major histocompatibility complex, and the small intestine. A molecular and immunobiologic approach to the spectrum of gluten sensitivity ('celiac sprue'). Gastroenterology 102, 330-354. doi: 10.1016/0016-5085(92)91819-P

Moher, D., Liberati, A., Tetzlaff, J., and Altman, D. G. (2009). Preferred reporting items for systematic reviews and meta-analyses: the PRISMA statement. BMJ 339:b2535. doi: 10.1136/bmj.b2535

Oberhuber, G., Granditsch, G., and Vogelsang, H. (1999). The histopathology of coeliac disease: time for a standardized report scheme for pathologists. Eur. J. Gastroenterol. Hepatol. 11, 1185-1194. doi: 10.1097/00042737-199910000-00019

Pekki, H., Kurppa, K., Maki, M., Huhtala, H., Laurila, K., Ilus, T., et al. (2017). Performing routine follow-up biopsy 1 year after diagnosis does not affect longterm outcomes in coeliac disease. Aliment. Pharmacol. Ther. 45, 1459-1468. doi: 10.1111/apt.14048

Pekki, H., Kurppa, K., Maki, M., Huhtala, H., Sievanen, H., Laurila, K., et al. (2015). Predictors and significance of incomplete mucosal recovery in celiac disease after 1 year on a gluten-free diet. Am. J. Gastroenterol. 110, 1078-1085. doi: 10.1038/ajg.2015.155 
Reilly, N. R., Husby, S., Sanders, D. S., and Green, P. H. R. (2017). Coeliac disease: to biopsy or not? Nat. Rev. Gastroenterol. Hepatol. 15:60-66. doi: 10.1038/nrgastro.2017.121

Rubio-Tapia, A., Hill, I. D., Kelly, C. P., Calderwood, A. H., and Murray, J. A. (2013). ACG clinical guidelines: diagnosis and management of celiac disease. Am. J. Gastroenterol. 108, 656-676. doi: 10.1038/ajg. 2013.79

Rubio-Tapia, A., Rahim, M. W., See, J. A., Lahr, B. D., Wu, T. T., and Murray, J. A. (2010). Mucosal recovery and mortality in adults with celiac disease after treatment with a gluten-free diet. Am. J. Gastroenterol. 105, 1412-1420. doi: 10.1038 /ajg.2010.10

Selby, W. S., Painter, D., Collins, A., Faulkner-Hogg, K. B., and Loblay, R. H. (1999). Persistent mucosal abnormalities in coeliac disease are not related to the ingestion of trace amounts of gluten. Scand. J. Gastroenterol. 34, 909-914. doi: 10.1080/003655299750025390

Souroujon, M., Ashkenazi, A., Lupo, M., Levin, S., and Hegesh, E. (1982). Serum ferritin levels in celiac disease. Am. J. Clin. Pathol. 77, 82-86. doi: $10.1093 /$ ajcp/77.1.82

Svedlund, J., Sjodin, I., and Dotevall, G. (1988). GSRS-a clinical rating scale for gastrointestinal symptoms in patients with irritable bowel syndrome and peptic ulcer disease. Dig. Dis. Sci. 33, 129-134. doi: 10.1007/BF015 35722

Szakacs, Z., Matrai, P., Hegyi, P., Szabo, I., Vincze, A., Balasko, M., et al. (2017). Younger age at diagnosis predisposes to mucosal recovery in celiac disease on a gluten-free diet: a meta-analysis. PLOS ONE 12:e0187526. doi: 10.1371/journal.pone. 0187526

Thompson, T. (1999). Thiamin, riboflavin, and niacin contents of the glutenfree diet: is there cause for concern? J. Am. Diet. Assoc. 99, 858-862. doi: 10.1016/S0002-8223(99)00205-9
Thornquist, H., Jacobsen, G. S., Dahl, L. B., and Marhaug, G. (1993) Coeliac disease and gluten-free diet: a following-up study of fifteen young adults. Ann. Nutr. Metab. 37, 295-301. doi: 10.1159/0001 77780

Tuire, I., Marja-Leena, L., Teea, S., Katri, H., Jukka, P., Paivi, S., et al. (2012). Persistent duodenal intraepithelial lymphocytosis despite a long-term strict gluten-free diet in celiac disease. Am. J. Gastroenterol. 107, 1563-1569. doi: 10.1038/ajg.2012.220

Valdimarsson, T., Toss, G., Ross, I., Lofman, O., and Strom, M. (1994). Bone mineral density in coeliac disease. Scand. J. Gastroenterol. 29, 457-461. doi: 10.3109/003655294090 96838

Walters, J. R., Banks, L. M., Butcher, G. P., and Fowler, C. R. (1995). Detection of low bone mineral density by dual energy $\mathrm{x}$ ray absorptiometry in unsuspected suboptimally treated coeliac disease. Gut 37, 220-224. doi: 10.1136/gut. 37.2 .220

Conflict of Interest: The authors declare that the research was conducted in the absence of any commercial or financial relationships that could be construed as a potential conflict of interest.

Copyright ( 2019 Szakács, Gede, Gyöngyi, Solymár, Csupor, Erőss, Vincze, Mikó, Vasas, Szapáry, Dobszai, Balikó, Hágendorn, Hegyi and Bajor. This is an open-access article distributed under the terms of the Creative Commons Attribution License (CC $B Y)$. The use, distribution or reproduction in other forums is permitted, provided the original author(s) and the copyright owner(s) are credited and that the original publication in this journal is cited, in accordance with accepted academic practice. No use, distribution or reproduction is permitted which does not comply with these terms. 